TRANSACTIONS OF THE

AMERICAN MATHEMATICAL SOCIETY

Volume 350, Number 12, December 1998, Pages 5017-5046

S $0002-9947(98) 02036-4$

\title{
DEGENERATE PRINCIPAL SERIES AND LOCAL THETA CORRESPONDENCE
}

\author{
SOO TECK LEE AND CHEN-BO ZHU
}

\begin{abstract}
In this paper we determine the structure of the natural $\widetilde{U}(n, n)$ module $\Omega^{p, q}(l)$ which is the Howe quotient corresponding to the determinant character $\operatorname{det}^{l}$ of $U(p, q)$. We first give a description of the tempered distributions on $M_{p+q, n}(\mathbb{C})$ which transform according to the character $\operatorname{det}^{-l}$ under the linear action of $U(p, q)$. We then show that after tensoring with a character, $\Omega^{p, q}(l)$ can be embedded into one of the degenerate series representations of $U(n, n)$. This allows us to determine the module structure of $\Omega^{p, q}(l)$. Moreover we show that certain irreducible constituents in the degenerate series can be identified with some of these representations $\Omega^{p, q}(l)$ or their irreducible quotients. We also compute the Gelfand-Kirillov dimensions of the irreducible constituents of the degenerate series.
\end{abstract}

\section{INTRODUCTION}

In his thesis $([\mathrm{L}])$, the first named author studied the module structure and unitarity of the following degenerate principal series representations of $U(n, n)$. Let $\left\{\varepsilon_{1}, \varepsilon_{2}, \ldots, \varepsilon_{2 n}\right\}$ be the standard basis of $\mathbb{C}^{2 n}$ and $\mathcal{B}$ be the basis $\left\{\varepsilon_{1}+\varepsilon_{n+1}, \ldots, \varepsilon_{n}+\right.$ $\left.\varepsilon_{2 n}, \varepsilon_{1}-\varepsilon_{n+1}, \ldots, \varepsilon_{n}-\varepsilon_{2 n}\right\}$. Let $S H(n)$ be the additive group of $n \times n$ skew-Hermitian matrices. For each $a \in G L(n, \mathbb{C})$ and $b \in S H(n)$, we let $m_{a}$ and $n_{b}$ be the elements of $U(n, n)$ with matrix representations $\left(\begin{array}{cc}a & 0 \\ 0 & \left(\bar{a}^{t}\right)^{-1}\end{array}\right)$ and $\left(\begin{array}{cc}I_{n} & b \\ 0 & I_{n}\end{array}\right)$ with respect to $\mathcal{B}$ respectively. Let

$$
M=\left\{m_{a}: a \in G L(n, \mathbb{C})\right\}, \quad N=\left\{n_{b}: b \in S H(n)\right\} .
$$

Then $P=M N$ is a parabolic subgroup of $U(n, n)$. For $s \in \mathbb{C}$ and $\nu \in \mathbb{Z}$, let $\chi_{s, \nu}$ be the character of $P$ given by

$$
\chi_{s, \nu}(p)=\chi_{s, \nu}\left(m_{a} n_{b}\right)=|\operatorname{det} a|^{s}\left(\frac{\operatorname{det} a}{|\operatorname{det} a|}\right)^{\nu}, \quad p=m_{a} n_{b} \in P .
$$

Let $I(s ; \nu)=\operatorname{Ind}_{P}^{U(n, n)} \chi_{s, \nu}$ be the corresponding (normalized) induced representation of $U(n, n)$. The representation space for $I(s ; \nu)$ is

$$
\left\{f \in C^{\infty}(U(n, n)): f(p g)=\chi_{s, \nu}(p) \Delta(p)^{\frac{1}{2}} f(g), \forall g \in U(n, n), p \in P\right\}
$$

where

$$
\Delta(p)=\Delta\left(m_{a} n_{b}\right)=|\operatorname{det} a|^{2 n}, \quad p=m_{a} n_{b} \in P,
$$

Received by the editors May 16, 1995 and, in revised form, January 27, 1997.

1991 Mathematics Subject Classification. Primary 22E46, 11F27.

Key words and phrases. degenerate principal series, local theta correspondence, Howe quotients, unitary representations, Gelfand-Kirillov dimension. 
is the modular function of $P$, and $U(n, n)$ acts on it by right translation:

$$
(g \cdot f)(h)=f(h g), \quad g, h \in U(n, n) .
$$

The first named author proved that $I(s ; \nu)$ is irreducible if and only if $\frac{s+n+\nu}{2} \notin$ $\mathbb{Z}$. Moreover in the case when $I(s ; \nu)$ is reducible, he obtained all its irreducible constituents.

Several other authors ([S1],[S2],[J1],[J2],[Zha]) have studied these representations in more general settings.

The purpose of this paper is two-fold. Firstly, we want to describe the irreducible constituents of $I(s ; \nu)$ more carefully in two ways. We consider the reductive dual pair

$$
(H, G)=(U(p, q), U(n, n)) \subseteq S p(4(p+q) n, \mathbb{R}) .
$$

Let $\widetilde{S} p(4(p+q) n, \mathbb{R})$ be the unique nontrivial double cover of $S p(4(p+q) n, \mathbb{R})$. For a subgroup $E$ of $S p(4(p+q) n, \mathbb{R}), \widetilde{E}$ shall denote the preimage of $E$ under the projection $\widetilde{S} p(4(p+q) n, \mathbb{R}) \longrightarrow \underset{\widetilde{S}}{S}(4(p+q) n, \mathbb{R})$. Let $V=\mathbb{C}^{p+q}$ and $V^{n}$ be the direct sum of $n$ copies of $V$. Then $\widetilde{S} p(4(p+q) n, \mathbb{R})$ acts on $L^{2}\left(V^{n}\right)$ via the oscillator representation $\omega$. We shall twist $\left.\omega\right|_{\overparen{H}}$ by a character so that it will factor through the standard linear action of $H$ on $L^{2}\left(V^{n}\right)$. Let $\mathcal{S}\left(V^{n}\right)$ be the Schwartz space of $V^{n}$. Let $S \subseteq \mathcal{S}\left(V^{n}\right)$ be the space of Schwartz functions which correspond to polynomials in the Fock model of $\omega$. For $l \in \mathbb{Z}$, let $D(l)$ be the irreducible $(\mathfrak{u}(p, q), U(p) \times U(q))$ module which corresponds to the determinant character $g \longrightarrow(\operatorname{det} g)^{l}$ of $U(p, q)$, and let $\Omega^{p, q}(l)$ be the maximal quotient of $S$ on which $(\mathfrak{u}(p, q), U(p) \times U(q))$ acts by a representation of class $D(l)$. They shall be referred to as the Howe quotients. We shall examine the relationship between the irreducible constituents in $I(s ; \nu)$ and these Howe quotients $\Omega^{p, q}(l)$. In particular, we shall show that some of the irreducible unitary submodules in $I(s ; \nu)$ can be identified with $\Omega^{p, q}(l)$. On the other hand we shall also measure the size of the irreducible constituents in $I(s ; \nu)$ by computing their Gelfand-Kirillov dimensions. In particular our results show that the Gelfand-Kirillov dimensions of the irreducible constituents can be read off from their position in the module diagram (or Hasse diagram, see $[\mathrm{L}]$ or $[\mathrm{Al}]$ ) of $I(s ; \nu)$. Our second purpose is to use the identification above and the results in [L] on the structure of the degenerate series to describe the module structure of the Howe quotients $\Omega^{p, q}(l)$. These results often play a role in the study of certain Eisenstein series and of $L$-functions attached to cuspidal automorphic representation for split groups ([KRS]).

This paper is arranged as follows. In section 2, we shall determine a set of all possible $\widetilde{K}$-types in $\Omega^{p, q}(l)$ and show that each of them occur with multiplicity at most one. Here $K=U(n) \times U(n)$ is a maximal compact subgroup of $U(n, n)$. In section 3 , we show that $\Omega^{p, q}(l)$ indeed contains these $\widetilde{K}$-types by considering the space $\left(\Omega^{*}\right)^{p, q}(l)$ of tempered distributions on $V^{n}$ which transform according to the character $\operatorname{det}^{-l}$ of $U(p, q)$. We show that as a $\widetilde{U}(n, n)$ module, $\left(\Omega^{*}\right)^{p, q}(l)$ is generated by a distinguished element $\mathcal{D}$ of $\left(\Omega^{*}\right)^{p, q}(l)$, and $\mathcal{D}$ has a non-zero projection to each of those $\widetilde{K}$-types which are the contragradient modules of the possible $\widetilde{K}$-types in $\Omega^{p, q}(l)$. This generalizes the results of the second named author on invariant tempered distributions [Zhu]. In section 4 , we shall twist $\Omega^{p, q}(l)$ by a character $\xi$ of $\widetilde{U}(n, n)$ and consider $\Omega_{\xi}^{p, q}(l)=\xi^{-1} \otimes \Omega^{p, q}(l)$. It turns out that $\Omega_{\xi}^{p, q}(l)$ factors through $U(n, n)$. We then use the element $\mathcal{D}$ to construct an embedding of 
$\Omega_{\xi}^{p, q}(l)$ into the degenerate series $\{I(s ; \nu): s \in \mathbb{C}, \nu \in \mathbb{Z}\}$ of $U(n, n)$ described at the beginning of this section.

Theorem 4.1. Let $m$ be a positive integer and let $p+q=m$. Assume either (i) $l=0$, or (ii) $l \neq 0$ and $m=n$. Then we have a $G$-equivariant embedding

$$
\lambda: \Omega_{\xi}^{p, q}(l) \hookrightarrow \begin{cases}I(m-n ; 0), & \text { if } l=0 \text { and } m \text { is even, } \\ I(m-n ;-m), & \text { if } l=0 \text { and } m \text { is odd, } \\ I(|l| ;-l), & \text { if } l \neq 0 \text { and } m=n \text { is even, } \\ I(|l| ;-n-l), & \text { if } l \neq 0 \text { and } m=n \text { is odd. }\end{cases}
$$

When $l \neq 0$, the $U(n, n)$ module $\Omega_{\xi}^{p, q}(l)$ is non-zero if and only if $m \leq n$, and in this case one can show that $\Omega_{\xi}^{p, q}(l)$ is irreducible and unitary. Also when $m<n$, it is impossible to embed $\Omega_{\xi}^{p, q}(l)$ into any $I(s ; \nu)$ (by comparing their $K$-types).

In section 5 , we use the results in sections 3 and 4 to identify the image of $\Omega_{\xi}^{p, q}(l)$ in $I(s ; \nu)$ under the above embedding. We then use the results of $[\mathrm{L}]$ to deduce the module structure of $\Omega_{\xi}^{p, q}(l)$. If $l=0$, we shall simply write $\Omega_{\xi}^{p, q}$ for $\Omega_{\xi}^{p, q}(0)$. Let $Q_{\xi}^{p, q}$ be the unique irreducible quotient of $\Omega_{\xi}^{p, q}$. The following theorem summarizes the results given in section 5 .

Theorem. Let

$$
(s, \nu)= \begin{cases}(m-n, 0), & \text { if } m \text { is even, } \\ (m-n,-m), & \text { if } m \text { is odd. }\end{cases}
$$

(a) If $1 \leq m \leq n$, then the set $\left\{\lambda\left(\Omega_{\xi}^{p, q}\right): p+q=m\right\}$ exhausts all the irreducible unitary submodules of $I(s ; \nu)$. Moreover, if $m=n$, then

$$
I(s ; \nu)=\bigoplus_{p+q=n} \lambda\left(\Omega_{\xi}^{p, q}\right) .
$$

(b) If $m \geq n+1$, then

$$
\lambda\left(\Omega_{\xi}^{p, q}\right)=M\left(d_{1}, d_{2}\right)
$$

and

$$
Q_{\xi}^{p, q} \cong R_{a\left(d_{1}, d_{2}\right)},
$$

where $d_{1}=\max \{0, n-q\}$ and $d_{2}=\max \{0, n-p\}$. Here $R_{a\left(d_{1}, d_{2}\right)}$ is an irreducible constituent of $I(s ; \nu)$ (see section 5 for its definition) and $M\left(d_{1}, d_{2}\right)$ is the submodule of $I(s ; \nu)$ generated by $R_{a\left(d_{1}, d_{2}\right)}$.

(c) $Q_{\xi}^{p, q}$ is unitary if and only if either $p \leq n$ and $q \leq n$, or $p q=0$. It is finite dimensional if and only if $p \geq n$ and $q \geq n$.

We also show that in the case when $l=0$ and $p+q \geq n$, all the constituents in the corresponding representation $I(s ; \nu)$ are determined by the images of the various Howe quotients $\Omega_{\xi}^{p, q}$. Since $I(s ; \nu)$ and $I(s ;-\nu)$ are contragradient, it follows that the images of Howe quotients determine all constituents of $I(s ; \nu)$ in general. Finally in section 6 , we compute the Gelfand-Kirillov dimensions of the irreducible constituents in $I(s ; \nu)$. 


\section{ACKNOWLEDGMENTS}

We would like to thank Professor Roger Howe and Professor Stephen Kudla for suggesting this problem to us and for their interest in this work. We would also like to thank the referee for many helpful suggestions. In particular he pointed out a gap in our original proof concerning the multiplicity-free property of $\left(\Omega^{*}\right)^{p, q}(l)$. The same gap also occurs in the proof of Theorem I of [Zhu].

\section{Howe's QUOTIENT $\Omega^{p, q}(l)$}

Consider the reductive dual pair $(H, G)=(U(p, q), U(n, n)) \subseteq S p(4(p+q) n, \mathbb{R})$. Let $L=U(p) \times U(q)$ and $K=U(n) \times U(n)$. Then $L$ and $K$ are maximal compact subgroups of $H$ and $K$, respectively. We shall choose a maximal compact subgroup $U \cong U(2(p+q) n)$ of $S p=S p(4(p+q) n, \mathbb{R})$ in such a way that $L=U \cap H$ and $K=$ $U \cap G$. We also let $\mathfrak{h}=\operatorname{Lie}(H)_{\mathbb{C}}$ and $\mathfrak{g}=\operatorname{Lie}(G)_{\mathbb{C}}$ be the complexified Lie algebras of the groups $H$ and $G$, respectively.

Let $V=\mathbb{C}^{p+q}$ and let $V^{n}$ be the direct sum of $n$ copies of $V$. Then $\widetilde{S} p(4(p+q) n, \mathbb{R})$ acts on $L^{2}\left(V^{n}\right)$ via the Oscillator representation $\omega$. As mentioned in the introduction, we can twist $\omega$ by a character so that it will factor through the following standard linear action of $H$ on $L^{2}\left(V^{n}\right)$ :

$$
h \cdot f\left(v_{1}, \ldots, v_{n}\right)=f\left(h^{-1} \cdot v_{1}, \ldots, h^{-1} \cdot v_{n}\right), \quad h \in H,\left(v_{1}, \ldots, v_{n}\right) \in V^{n} .
$$

We shall assume that this has been done from now on. Let $\mathcal{S}\left(V^{n}\right)$ be the Schwartz space of $V^{n}$ and let $S$ be the space consisting of those Schwartz functions which correspond to polynomials in a Fock model of $\omega$. Since $S$ is the space of $\widetilde{U}$-finite vectors of $\omega$, it is naturally a $(\mathfrak{h}, L) \times(\mathfrak{g}, \widetilde{K})$-module.

For any integer $l$, we let $D(l)$ be the irreducible $(\mathfrak{h}, L)$ module corresponding to the character $h \longrightarrow(\operatorname{det} h)^{l}$ of $U(p, q)$. Let $\Omega^{p, q}(l)$ be the maximal quotient of $S$ on which $(\mathfrak{h}, L)$ acts by a representation of class $D(l)$. By the results in $[\mathrm{H} 2], \Omega^{p, q}(l)$ is a quasi-simple $(\mathfrak{g}, \widetilde{K})$-module of finite length and has a unique irreducible quotient $Q^{p, q}(l)$. Note that $Q^{p, q}(l)$ is the representation of $(\mathfrak{g}, \widetilde{K})$ which corresponds to the character $\operatorname{det}^{l}$ of $U(p, q)$ under Howe's quotient correspondence.

In [KR1], Kudla and Rallis consider the dual pair

$$
(O(p, q), S p(2 n, \mathbb{R})) \subseteq S p(2(p+q) n, \mathbb{R})
$$

and study the Howe's quotient $R$ which corresponds to the trivial representation of $O(p, q)$. In particular, they give the $\widetilde{U}(n)$-spectrum of $R$, which is multiplicity free. In the present case, we shall show that the $\widetilde{K}$-spectrum of $\Omega^{p, q}(l)$ is also multiplicity free.

We need to introduce some notation. Let $\Lambda_{n}^{+}$be the set of all dominant integral weights for the unitary group $U(n)$ with respect to the Borel subalgebra $\mathfrak{b}_{n}^{+}$of upper triangular matrices. $\Lambda_{n}^{+}$can be identified in the usual way with the set of all $n$-tuples of integers $\lambda=\left(\lambda_{1}, \lambda_{2}, \ldots, \lambda_{n}\right)$ such that $\lambda_{1} \geq \lambda_{2} \geq \ldots \geq \lambda_{n}$. For each $\lambda \in \Lambda_{n}^{+}$, $\rho^{\lambda}$ shall denote a copy of the irreducible representation of $U(n)$ with highest weight $\lambda$. Recall that if $\rho=\rho^{\lambda}$, then the contragradient of $\rho$ is given by $\rho^{*}=\rho^{\lambda^{*}}$, where $\lambda^{*}=\left(-\lambda_{n}, \ldots,-\lambda_{1}\right)$. For convenience, we also let

$$
\mathbf{1}_{n}=(1, \ldots, 1) .
$$


Note that $\mathbf{1}_{n}$ is the highest weight of the determinant character of $U(n)$. In a similar way, every irreducible representation of $\widetilde{U}(n)$ is of the form $\rho^{\lambda}$, but the components of $\lambda$ can be half integers.

Proposition 2.1. Every $\widetilde{K}$-type $\tau$ in $\Omega^{p, q}(0)$ is of the form

$$
\tau \cong \rho^{\lambda} \otimes \rho^{\lambda^{*}}
$$

where

$$
\lambda=\frac{p-q}{2} \mathbf{1}_{n}+\left(\alpha_{1}, \ldots, \alpha_{t}, 0, \ldots, 0,-\gamma_{s}, \ldots,-\gamma_{1}\right),
$$

and $\alpha_{1} \geq \ldots \geq \alpha_{t} \geq 0, \gamma_{1} \geq \ldots \geq \gamma_{s} \geq 0$ are integers with $t \leq \min (p, n)$ and $s \leq \min (q, n)$. Moreover each such $\widetilde{K}$-type occurs with multiplicity at most one.

Proposition 2.2. For $l \neq 0, \Omega^{p, q}(l)$ is nontrivial only if $p+q \leq n$. If $p+q \leq n$, then every $\widetilde{K}$-type $\tau$ in $\Omega^{p, q}(l)$ is of the form

$$
\tau \cong \rho^{\lambda} \otimes \rho^{\lambda^{*}}+\overbrace{-l, \ldots,-l, 0, \ldots, 0,0,-l, \ldots,-l}^{q \text { times }},
$$

where

$$
\lambda=\frac{p-q}{2} \mathbf{1}_{n}+\left(\alpha_{1}, \ldots, \alpha_{p}, 0, \ldots, 0,-\gamma_{q}, \ldots,-\gamma_{1}\right),
$$

and $\alpha_{i}, \gamma_{i}$ are integers satisfying

$$
\alpha_{1} \geq \ldots \geq \alpha_{p} \geq 0, \gamma_{1} \geq \ldots \geq \gamma_{q} \geq l, \quad \text { if } l>0,
$$

and

$$
\alpha_{1} \geq \ldots \geq \alpha_{p} \geq-l, \gamma_{1} \geq \ldots \geq \gamma_{q} \geq 0, \quad \text { if } l<0 .
$$

Moreover each such $\widetilde{K}$-type occurs with multiplicity at most one.

Proof of Propositions 2.1 and 2.2. The argument given below is similar to those of [KR1] in the case of dual pair $(O(p, q), S p(2 n, \mathbb{R}))$. We consider the seesaw dual pair $([\mathrm{Ku} 1])$

$$
\begin{array}{cc}
U(p, q) & U(n) \times U(n) \\
\cap & \cap \\
U(p, q) \times U(p, q) & U(n, n) .
\end{array}
$$

Assume that $\tau=\rho^{\lambda} \otimes \rho^{\mu}$ is a $\widetilde{K}$-type occurring in $\Omega^{p, q}(l)$. As usual we use a subscript $\tau$ to denote the $\tau$-isotypic component. By the standard result of Howe $([\mathrm{H} 2])$ or Kashiwara and Vergne $([\mathrm{KV}])$, we have

$$
S_{\tau} \cong \pi(\tau) \otimes \tau
$$

for some irreducible $\left(\mathfrak{u}(p, q) \oplus \mathfrak{u}(p, q), K^{\prime}\right)$-module $\pi(\tau)$, where $K^{\prime}=U(p) \times U(q) \times$ $U(p) \times U(q)$. Let $\mathcal{H}(K)$ be the space of $K$-harmonics of $S$. Then we have $\mathcal{H}(K)_{\tau} \cong$ $\sigma(\tau) \otimes \tau$, where $\sigma(\tau)=\sigma_{1} \otimes \sigma_{3} \otimes \sigma_{2} \otimes \sigma_{4} \in \widehat{K^{\prime}}, \sigma_{1}, \sigma_{2} \in \widehat{U}(p)$ and $\sigma_{3}, \sigma_{4} \in \widehat{U}(q)$, and $\sigma(\tau)$ occurs in $\pi(\tau)$ with multiplicity one.

The arguments in [KR1] give

$$
\Omega^{p, q}(l)_{\tau}=\operatorname{pr}\left(S_{\tau}\right)=\operatorname{pr}\left(\mathcal{H}(K)_{\tau}\right),
$$


where $p r: S \rightarrow \Omega^{p, q}(l)$ is the natural projection map. Now the projection $p r: \mathcal{H}(K)_{\tau} \rightarrow \Omega^{p, q}(l)_{\tau}$ factors through the projection $\mathcal{H}(K)_{\tau} \rightarrow \mathcal{H}(K)_{\tau, l}$, where $\mathcal{H}(K)_{\tau, l}$ consists of those $v \in \mathcal{H}(K)_{\tau}$ such that

$$
h \cdot v=\left(\operatorname{det} h_{1}\right)^{l}\left(\operatorname{det} h_{2}\right)^{l} v, \quad h=\left(h_{1}, h_{2}\right) \in U(p) \times U(q) .
$$

Recall that we have twisted the oscillator representation $\omega$ by a character so that $H=U(p, q)$ acts linearly. Thus $U(p) \times U(q)$ also acts linearly on $\mathcal{H}(K)$. Taking this into consideration, the results of $[\mathrm{KV}]$ imply that $\tau=\rho^{\lambda} \otimes \rho^{\mu}$ has the form

$$
\begin{aligned}
\lambda & =\frac{p-q}{2} \mathbf{1}_{n}+\left(\alpha_{1}, \ldots, \alpha_{t}, 0, \ldots, 0,-\gamma_{s}, \ldots,-\gamma_{1}\right), \\
\mu & =-\frac{p-q}{2} \mathbf{1}_{n}+\left(\delta_{1}, \ldots, \delta_{s}, 0, \ldots, 0,-\beta_{t}, \ldots,-\beta_{1}\right),
\end{aligned}
$$

where $t \leq \min (p, n), s \leq \min (q, n), \alpha_{i}, \beta_{i}, \gamma_{i}, \delta_{i}$ are non-negative integers, and

$$
\begin{aligned}
& \sigma_{1} \otimes \sigma_{2}=\rho^{\left(0, \ldots, 0,-\alpha_{t}, \ldots,-\alpha_{1}\right)} \otimes \rho^{\left(\beta_{1}, \ldots, \beta_{t}, 0, \ldots, 0\right)} \in \widehat{K_{1}^{\prime}}, \\
& \sigma_{3} \otimes \sigma_{4}=\rho^{\left(\gamma_{1}, \ldots, \gamma_{s}, 0, \ldots, 0\right)} \otimes \rho^{\left(0, \ldots, 0,-\delta_{s}, \ldots,-\delta_{1}\right)} \in \widehat{K_{2}^{\prime}},
\end{aligned}
$$

where $K_{1}^{\prime}=U(p) \times U(p), K_{2}^{\prime}=U(q) \times U(q)$.

Hence for $\Omega^{p, q}(l)_{\tau} \neq 0$, the representation $\sigma(\tau)=\sigma_{1} \otimes \sigma_{3} \otimes \sigma_{2} \otimes \sigma_{4}$ must contain the character $\operatorname{det}_{p}^{l} \otimes \operatorname{det}_{q}^{l}$ of $U(p) \times U(q)$. This occurs when $\sigma_{2} \cong \sigma_{1}^{*} \otimes \operatorname{det}_{p}^{l}$, and $\sigma_{4} \cong \sigma_{3}^{*} \otimes \operatorname{det}_{q}^{l} ;$ i.e.,

$$
\begin{aligned}
& \beta_{i}=\alpha_{i}, \delta_{i}=\gamma_{i}, \text { if } l=0, \\
& \beta_{i}=\alpha_{i}+l, \delta_{i}=\gamma_{i}-l, t=p, s=q, \text { if } l \neq 0 .
\end{aligned}
$$

Moreover when the above conditions are satisfied, $\sigma(\tau)$ contains the character $\operatorname{det}_{p}^{l} \otimes \operatorname{det}_{q}^{l}$ exactly once. For $l \neq 0$, the second condition above implies that $p+q \leq n, \gamma_{i} \geq l$ if $l>0$, and $\alpha_{i} \geq-l$ if $l<0$. Further we have

$$
\mu=-\frac{p-q}{2} \mathbf{1}_{n}+\left(\gamma_{1}, \ldots, \gamma_{s}, 0, \ldots, 0,-\alpha_{t}, \ldots,-\alpha_{1}\right)+(\underbrace{-l, \ldots,-l}_{q \text { times }}, 0, \ldots, 0, \underbrace{-l, \ldots, l}_{p \text { times }}) .
$$

Thus $\mu=\lambda^{*}+(\underbrace{-l, \ldots,-l}_{q \text { times }}, 0, \ldots, 0, \underbrace{-l, \ldots,-l}_{p \text { times }})$.

We shall show in the next section that these $\widetilde{K}$-types indeed occur in $\Omega^{p, q}(l)$ by considering a certain space $\left(\Omega^{*}\right)^{p, q}(l)$ of tempered distributions.

\section{3. $U(p, q)$-EQUIVARIANT TEMPERED DISTRIBUTIONS}

Recall that $\mathcal{S}\left(V^{n}\right)$ is the Schwartz space of $V^{n}$, where $V$ is the standard module of $H=U(p, q)$. Let $\mathcal{S}^{*}\left(V^{n}\right)$ be the space of tempered distributions on $V^{n}$. Then $\widetilde{S} p(4(p+q) n, \mathbb{R})$ acts on $\mathcal{S}\left(V^{n}\right)$ by the restriction of the oscillator representation $\omega$ and this action induces an action of $\widetilde{S} p(4(p+q) n, \mathbb{R})$ on $\mathcal{S}^{*}\left(V^{n}\right)$ in the usual way. We shall denote this action on $\mathcal{S}^{*}\left(V^{n}\right)$ also by $\omega$.

Now for each integer $l$, let $\left(\Omega^{*}\right)^{p, q}(l)$ be the subspace of tempered distributions consisting of those $\Phi \in \mathcal{S}^{*}\left(V^{n}\right)$ such that

$$
h \cdot \Phi=(\operatorname{det} h)^{-l} \Phi, \quad h \in H,
$$

where $h \cdot \Phi=\omega(h) \Phi$ is the linear action of $H$ on $\mathcal{S}^{*}\left(V^{n}\right)$. 
Since the action of $\widetilde{G}$ commutes with the action of $H,\left(\Omega^{*}\right)^{p, q}(l)$ is a $\widetilde{G}$ module. When $l=0,\left(\Omega^{*}\right)^{p, q}(0)=\mathcal{S}^{*}\left(V^{n}\right)^{H}$ is the space of $H$-invariant tempered distributions on $V^{n}$. A precise description of this representation is given in [Zhu] and we shall recall it in Theorem 3.1 below for the convenience of the readers. The purpose of this section is to show that for $l \neq 0$, the $\widetilde{G}$ module $\left(\Omega^{*}\right)^{p, q}(l)$ is generated by a certain tempered distribution $\mathcal{D}$ and to determine the $\widetilde{K}$-types in $\left(\Omega^{*}\right)^{p, q}(l)$.

As usual, $\delta$ shall denote the Dirac distribution at the origin of $V^{n}$. It is $H$ invariant.

Theorem 3.1 (Theorem, $[\mathrm{Zhu}]$ ). (a) $\mathcal{S}^{*}\left(V^{n}\right)^{H}$ is the closed span of the set $\{\omega(g) \delta$ : $g \in \widetilde{G}\}$.

(b) For any $\sigma \in \widehat{\widetilde{K}}$, the multiplicity of $\sigma$ in $\mathcal{S}^{*}\left(V^{n}\right)^{H}$ is at most one. It is equal to one if and only if

$$
\sigma^{*} \cong \rho^{\lambda} \otimes \rho^{\lambda^{*}}
$$

where

$$
\lambda=\frac{p-q}{2} \mathbf{1}_{n}+\left(\alpha_{1}, \ldots, \alpha_{t}, 0, \ldots, 0,-\gamma_{s}, \ldots,-\gamma_{1}\right),
$$

and

$$
\alpha_{1} \geq \ldots \geq \alpha_{t} \geq 0, \quad \gamma_{1} \geq \ldots \geq \gamma_{s} \geq 0
$$

are integers and $t \leq \min (p, n)$ and $s \leq \min (q, n)$.

Remark 3.2. Theorem I of [Zhu] states that the $\widetilde{K}$-types in $\mathcal{S}^{*}\left(V^{n}\right)$ are of the form $\sigma \cong \rho^{\lambda} \otimes \rho^{\lambda^{*}}$, where $\lambda$ is given above. This is incorrect (see Theorem 3.3 below). A proof for the corrected version can be obtained by making only a minor change in the original proof (see the proof of Theorem 3.3 below).

Now we shall examine the space $\left(\Omega^{*}\right)^{p, q}(l)$ for $l \neq 0$. We shall introduce a distinguished tempered distribution in $\left(\Omega^{*}\right)^{p, q}(l)$, which will take the place of $\delta$ in $\Omega^{*}(0)$. Let $Z=\left(z_{i j}\right)_{1 \leq i \leq p+q, 1 \leq j \leq n}$ be the natural complex coordinates of $V^{n} \cong$ $M_{p+q, n}(\mathbb{C})$. For $1 \leq t \leq \min (p+q, n)$, let

$$
d_{t}(Z)=\operatorname{det}\left(\begin{array}{ccc}
z_{11} & \ldots & z_{1 t} \\
\ldots & \ldots & \ldots \\
z_{t 1} & \ldots & z_{t t}
\end{array}\right), \bar{d}_{t}(Z)=\operatorname{det}\left(\begin{array}{ccc}
\bar{z}_{11} & \ldots & \bar{z}_{1 t} \\
\ldots & \ldots & \ldots \\
\bar{z}_{t 1} & \ldots & \bar{z}_{t t}
\end{array}\right)
$$

$$
\partial_{t}=\partial_{t}(Z)=\operatorname{det}\left(\begin{array}{ccc}
\frac{\partial}{\partial z_{11}} & \cdots & \frac{\partial}{\partial z_{1 t}} \\
\cdots & \cdots & \cdots \\
\frac{\partial}{\partial z_{t 1}} & \cdots & \frac{\partial}{\partial z_{t t}}
\end{array}\right), \quad \bar{\partial}_{t}=\bar{\partial}_{t}(Z)=\operatorname{det}\left(\begin{array}{ccc}
\frac{\partial}{\partial \bar{z}_{11}} & \cdots & \frac{\partial}{\partial \bar{z}_{1 t}} \\
\cdots & \cdots & \cdots \\
\frac{\partial}{\partial \bar{z}_{t 1}} & \cdots & \frac{\partial}{\partial \bar{z}_{t t}}
\end{array}\right) .
$$

Assume that $p+q \leq n$ and $d>0$. Then one can verify that for $h \in H$, we have

$$
\begin{aligned}
& h \cdot\left(\partial_{p+q}^{d} \delta\right)=(\operatorname{det} h)^{d}\left(\partial_{p+q}^{d} \delta\right), \\
& h \cdot\left(\bar{\partial}_{p+q}^{d} \delta\right)=(\operatorname{det} h)^{-d}\left(\bar{\partial}_{p+q}^{d} \delta\right) .
\end{aligned}
$$

Consequently the tempered distribution $\mathcal{D}$ given by

$$
\mathcal{D}= \begin{cases}\bar{\partial}_{p+q}^{l} \delta, & \text { if } l>0, \\ \partial_{p+q}^{-l} \delta, & \text { if } l<0,\end{cases}
$$

is in the space $\left(\Omega^{*}\right)^{p, q}(l)$. 
Theorem 3.3. For $l \neq 0,\left(\Omega^{*}\right)^{p, q}(l)$ is nontrivial if and only if $p+q \leq n$. If $p+q \leq n$, then

(a) $\left(\Omega^{*}\right)^{p, q}(l)$ is the closed span of the set $\{\omega(g) \mathcal{D} \mid g \in \widetilde{G}\}$.

(b) For any $\sigma \in \widehat{\widetilde{K}}$, the multiplicity of $\sigma$ in $\left(\Omega^{*}\right)^{p, q}(l)$ is at most one. It is equal to one if and only if

$$
\sigma^{*} \cong \rho^{\lambda} \otimes \rho^{\lambda^{*}}+(\overbrace{-l, \ldots,-l, 0, \ldots, 0,-l, \ldots,-l}^{\text {times }}),
$$

where

$$
\lambda=\frac{p-q}{2} \mathbf{1}_{n}+\left(\alpha_{1}, \ldots, \alpha_{p}, 0, \ldots, 0,-\gamma_{q}, \ldots,-\gamma_{1}\right),
$$

and $\alpha_{i}, \gamma_{i}$ are integers satisfying

$$
\alpha_{1} \geq \ldots \geq \alpha_{p} \geq 0, \gamma_{1} \geq \ldots \geq \gamma_{q} \geq l, \quad \text { if } l>0,
$$

and

$$
\alpha_{1} \geq \ldots \geq \alpha_{p} \geq-l, \gamma_{1} \geq \ldots \geq \gamma_{q} \geq 0, \quad \text { if } l<0
$$

We outline our strategy for proving Theorems 3.1 and 3.3, and we thank the referee for helping us to clarify this. Let $R^{p, q}(l)$ be the maximal quotient of $\mathcal{S}\left(V^{n}\right)$ on which $U(p, q)$ acts by the character $g \rightarrow(\operatorname{det} g)^{l}$. Clearly $R^{p, q}(l)$ and $\left(\Omega^{*}\right)^{p, q}(l)$ are duals under the natural pairing \langle\rangle between $\mathcal{S}\left(V^{n}\right)$ and $\mathcal{S}^{*}\left(V^{n}\right)$. There is also a natural map $\eta: \Omega^{p, q}(l) \mapsto R^{p, q}(l)$, which makes the following diagram commutative:

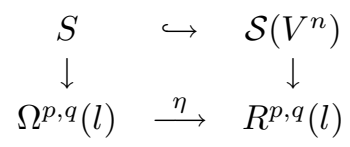

Here the vertical maps are the natural quotient maps. If $\tau$ is a possible $\widetilde{K}$-type in $\Omega^{p, q}(l)$ (as given in Propositions 2.1 and 2.2), we shall prove that there exists a vector $\phi \in S_{\tau}$ such that $\langle\mathcal{D}, \phi\rangle \neq 0$. Since $\mathcal{D} \in\left(\Omega^{*}\right)^{p, q}(l)$, we see that the image of $\phi$ is non-zero in $R^{p, q}(l)$, and since the map of $\phi$ to this space factors through $\Omega^{p, q}(l)$, the image of $\phi$ in $\Omega^{p, q}(l)$ is also non-zero. Thus (i) all such $\tau^{\prime}$ s occur in $\Omega^{p, q}(l)$, and (ii) since $\Omega^{p, q}(l)$ is $\widetilde{K}$-multiplicity free, the map $\eta$ is injective. Now since the space $S$ is dense in the Fréchet space $\mathcal{S}\left(V^{n}\right)$, we see $\eta\left(\Omega^{p, q}(l)\right)$ is dense in $R^{p, q}(l)$, and by the injectivity of $\eta$, we obtain a non-degenerate pairing between $\Omega^{p, q}(l)$ and $\left(\Omega^{*}\right)^{p, q}(l)$, which is $\widetilde{K}$-equivariant. We can then conclude that $\widetilde{K}$-types of $\left(\Omega^{*}\right)^{p, q}(l)$ must be contragradient to the $\widetilde{K}$-types of $\Omega^{p, q}(l)$, and are thus of the form given in Theorems 3.1 and 3.3, with their $\widetilde{K}$-multiplicities equal to one, and each $\widetilde{K}$-isotypic component of $\left(\Omega^{*}\right)^{p, q}(l)$ is generated as a $\widetilde{K}$-module by the corresponding $\widetilde{K}$-isotypic component of $\mathcal{D}$. This will imply Theorems 3.1 and 3.3 .

Let $\omega^{-\infty}$ be the space of formal vectors on $\omega$ ([HT], [Zhu]). It is the space of generalized functions on $V^{n}$ with $\widetilde{U}$ Fourier components, where $U \cong U(2(p+q) n)$ is a maximal compact subgroup of $S p(4(p+q) n, \mathbb{R})$. Moreover, $\mathcal{S}\left(V^{n}\right)\left(\operatorname{resp} . \mathcal{S}^{*}\left(V^{n}\right)\right)$ can be characterized as the subspace of $\omega^{-\infty}$ such that their $\widetilde{U}$ Fourier components decay rapidly (resp. grow at most polynomially). Notice that $\widetilde{S} p(4(p+q) n, \mathbb{R})$ acts on $\omega^{-\infty}$ by linear extension from the action on $\mathcal{S}\left(V^{n}\right)$ and thus it is contragradient 
to the action on $\mathcal{S}^{*}\left(V^{n}\right)$. The main purpose in introducing the space $\omega^{-\infty}$ is that the distribution $\mathcal{D}$ turns out to have a simple expression as an formal vector, and that simple expression enables us to compute its inner product with certain lowest highest weight vectors. We shall first examine the compact case.

Proposition 3.4. (a) Suppose that $H=U(p, 0)=U(p)$ and $p \leq n$. Let $\tau \in \widehat{\widetilde{K}}$ be such that

$$
\tau \cong \rho^{\lambda} \otimes \rho{ }^{\lambda^{*}+(0, \ldots, 0, \underbrace{-l, \ldots,-l}_{p \text { times }})}
$$

where $\lambda=\frac{p}{2} \mathbf{1}_{n}+\left(\alpha_{1}, \alpha_{2}, \ldots, \alpha_{p}, 0, \ldots, 0\right)$, and $\alpha_{i}$ are integers satisfying

$$
\alpha_{1} \geq \ldots \geq \alpha_{p} \geq 0, \quad \text { if } l>0,
$$

and

$$
\alpha_{1} \geq \ldots \geq \alpha_{p} \geq-l, \quad \text { if } l<0 .
$$

Then there exists a vector $\phi \in S_{\tau}$ such that $\langle\mathcal{D}, \phi\rangle \neq 0$. In particular, $\mathcal{D}$ has a non-zero $\sigma$-isotypic component for $\sigma \cong \tau^{*}$.

(b) Suppose $H=U(0, q)$ and $q \leq n$. Let $\tau \in \widehat{\widetilde{K}}$ be such that

$$
\tau=\rho^{\lambda} \otimes \rho{ }_{q \text { times }}^{\lambda^{*}+(\underbrace{-l, \ldots,-l}, 0, \ldots, 0)},
$$

where $\lambda=-\frac{q}{2} \mathbf{1}_{n}+\left(0, \ldots, 0,-\gamma_{q}, \ldots,-\gamma_{1}\right)$, and $\gamma_{i}$ are integers satisfying

$$
\gamma_{1} \geq \ldots \geq \gamma_{q} \geq l, \quad \text { if } l>0,
$$

and

$$
\gamma_{1} \geq \ldots \geq \gamma_{q} \geq 0, \quad \text { if } l<0
$$

Then there exists a vector $\phi \in S_{\tau}$ such that $\langle\mathcal{D}, \phi\rangle \neq 0$. In particular, $\mathcal{D}$ has a non-zero $\sigma$-isotypic component for $\sigma \cong \tau^{*}$.

Proof. We shall only prove part (a). The proof for part (b) is similar. Recall $Z=\left(z_{i j}\right)_{1 \leq i \leq p, 1 \leq j \leq n}$ are the standard coordinates of $V^{n} \cong M_{p, n}(\mathbb{C})$. Let $Q=$ $\left(q_{i j}\right)_{1 \leq i \leq p, 1 \leq j \leq n}$ and $\bar{Q}=\left(\bar{q}_{i j}\right)_{1 \leq i \leq p, 1 \leq j \leq n}$ be the complex coordinates in the Fock model of the oscillator representation of $\omega$. The isometric isomorphism of the Schrödinger model with the Fock model is such that (cf. [Fo])

$$
\begin{aligned}
& z_{i j} \longrightarrow \frac{1}{\sqrt{2}}\left(2 \frac{\partial}{\partial \bar{q}_{i j}}+q_{i j}\right), \frac{\partial}{\partial z_{i j}} \longrightarrow \frac{1}{2 \sqrt{2}}\left(2 \frac{\partial}{\partial q_{i j}}-\bar{q}_{i j}\right), \\
& \bar{z}_{i j} \longrightarrow \frac{1}{\sqrt{2}}\left(2 \frac{\partial}{\partial q_{i j}}+\bar{q}_{i j}\right), \frac{\partial}{\partial \bar{z}_{i j}} \longrightarrow \frac{1}{2 \sqrt{2}}\left(2 \frac{\partial}{\partial \bar{q}_{i j}}-q_{i j}\right) .
\end{aligned}
$$

Let $\Delta$ be the image of $\delta$, the Dirac distribution at the origin of $V^{n}$. Since $\delta$ satisfies

$$
z_{i j} \cdot \delta=\bar{z}_{i j} \cdot \delta=0
$$

we have

$$
\Delta=\exp \left(-\frac{\sum_{i j} q_{i j} \bar{q}_{i j}}{2}\right) \quad(\text { up to a scalar })
$$

as an element in $\omega^{-\infty}$.

Recall the definitions of $d_{t}(Z), \bar{d}_{t}(Z), \partial_{t}(Z), \bar{\partial}_{t}(Z)$ as given in eqs. (3.1), (3.2). We define $d_{t}(Q), \bar{d}_{t}(Q), \partial_{t}(Q), \bar{\partial}_{t}(Q)$ by replacing $z_{i j}$ with $q_{i j}$ in $d_{t}(Z), \bar{d}_{t}(Z)$, $\partial_{t}(Z)$ and $\bar{\partial}_{t}(Z)$ respectively. 
For any operator $A$, let $A^{*}$ be its adjoint with respect to the Hilbert space structure in the Fock model. We have (cf. [Ba])

$$
\left(\frac{\partial}{\partial q_{i j}}\right)^{*}=\frac{1}{2} q_{i j},\left(\frac{\partial}{\partial \bar{q}_{i j}}\right)^{*}=\frac{1}{2} \bar{q}_{i j} .
$$

Thus

$$
\partial_{t}(Q)^{*}=\frac{1}{2^{t}} d_{t}(Q), \bar{\partial}_{t}(Q)^{*}=\frac{1}{2^{t}} \bar{d}_{t}(Q) .
$$

Under the isomorphism from the Schrödinger model to the Fock model, a tempered distribution $\Phi \in \mathcal{S}^{*}\left(V^{n}\right)$ is identified with a formal vector $\mathcal{F}_{\Phi} \in \omega^{-\infty}$. If a polynomial coefficient differential operator $B$ in $z_{i j}, \bar{z}_{i j}$ is identified with a polynomial coefficient differential operator $\mathcal{F}_{B}$ in $q_{i j}, \bar{q}_{i j}$, then the distribution $B \cdot \Phi$ with be identified with the formal vector $-\left(\mathcal{F}_{B}\right)^{*} \cdot \mathcal{F}_{\Phi}$, in view of the fact that $\widetilde{S} p(4(p+q) n, \mathbb{R}) \ltimes H$ acts on $\omega^{-\infty}$ by linear extension from the action on $\mathcal{S}\left(V^{n}\right)$, which is contragradient to the action on $\mathcal{S}^{*}\left(V^{n}\right)$. Here $H$ is the standard Heisenberg group associated to the symplectic group $S p(4(p+q) n, \mathbb{R})$. This implies that

$$
\begin{gathered}
\frac{\partial}{\partial z_{i j}} \delta \longrightarrow-\frac{1}{2 \sqrt{2}}\left(2 \frac{\partial}{\partial q_{i j}}-\bar{q}_{i j}\right)^{*} \exp \left(-\frac{\sum_{i j} q_{i j} \bar{q}_{i j}}{2}\right)=\frac{1}{\sqrt{2}} \frac{\partial}{\partial \bar{q}_{i j}} \exp \left(-\frac{\sum_{i j} q_{i j} \bar{q}_{i j}}{2}\right), \\
\frac{\partial}{\partial \bar{z}_{i j}} \delta \longrightarrow-\frac{1}{2 \sqrt{2}}\left(2 \frac{\partial}{\partial \bar{q}_{i j}}-q_{i j}\right)^{*} \exp \left(-\frac{\sum_{i j} q_{i j} \bar{q}_{i j}}{2}\right)=\frac{1}{\sqrt{2}} \frac{\partial}{\partial q_{i j}} \exp \left(-\frac{\sum_{i j} q_{i j} \bar{q}_{i j}}{2}\right) .
\end{gathered}
$$

Thus we have

$$
\begin{aligned}
& \partial_{t}(Z) \delta \longrightarrow\left(\frac{1}{\sqrt{2}}\right)^{t} \bar{\partial}_{t}(Q) \Delta, \\
& \bar{\partial}_{t}(Z) \delta \longrightarrow\left(\frac{1}{\sqrt{2}}\right)^{t} \partial_{t}(Q) \Delta,
\end{aligned}
$$

and so the formal vector $F_{\mathcal{D}}$ corresponding to $\mathcal{D}$ is

$$
F_{\mathcal{D}}= \begin{cases}\left(\frac{1}{\sqrt{2}}\right)^{p l} \partial_{p}(Q)^{l} \Delta, & l>0 \\ \left(\frac{1}{\sqrt{2}}\right)^{p l} \bar{\partial}_{p}(Q)^{-l} \Delta, & l<0\end{cases}
$$

In the Fock model, a highest weight vector in the $\widetilde{K}$-type

$$
\tau=\rho^{\lambda} \otimes \rho^{\mu}=\rho^{\frac{p}{2} \mathbf{1}_{n}+\left(\alpha_{1}, \ldots, \alpha_{t}, 0, \ldots, 0\right)} \otimes \rho^{-\frac{p}{2} \mathbf{1}_{n}+\left(0, \ldots, 0,-\beta_{t}, \ldots,-\beta_{1}\right)}, \quad t \leq p,
$$

is given by

$$
d_{1}(Q)^{a_{1}} d_{2}(Q)^{a_{2}} \ldots d_{t}(Q)^{a_{t}} \bar{d}_{1}(Q)^{b_{1}} \bar{d}_{2}(Q)^{b_{2}} \ldots \bar{d}_{t}(Q)^{b_{t}}
$$

where

$$
\alpha_{i}=\sum_{j=i}^{t} a_{j}, \quad \beta_{i}=\sum_{j=i}^{t} b_{j}
$$

We may assume that $t=p$ by allowing $\alpha_{i}, \beta_{i}$ to be zero.

Now the condition that $\mu=\lambda^{*}+(0, \ldots, 0, \underbrace{-l, \ldots,-l}_{p \text { times }})$ amounts to $\beta_{i}=\alpha_{i}+l$, $\forall i \leq p$, i.e.,

$$
\begin{aligned}
b_{i} & =a_{i}, \quad 1 \leq i \leq p-1 \\
b_{p} & =a_{p}+l
\end{aligned}
$$


We first consider the case $l>0$. We compute the following inner product of $F_{\mathcal{D}}$ with a $\widetilde{K}$ highest weight vector (using the adjoint relation (3.4)):

$$
\begin{aligned}
& \left(\partial_{p}(Q)^{l} \Delta, d_{1}(Q)^{a_{1}} d_{2}(Q)^{a_{2}} \ldots d_{p}(Q)^{a_{p}} \bar{d}_{1}(Q)^{b_{1}} \bar{d}_{2}(Q)^{b_{2}} \ldots \bar{d}_{p}(Q)^{b_{p}}\right) \\
& =\left(\frac{1}{2^{p}}\right)^{l}\left(\Delta, d_{p}(Q)^{l} \cdot d_{1}(Q)^{a_{1}} d_{2}(Q)^{a_{2}} \ldots d_{p}(Q)^{a_{p}} \bar{d}_{1}(Q)^{b_{1}} \bar{d}_{2}(Q)^{b_{2}} \ldots \bar{d}_{p}(Q)^{b_{p}}\right) \\
& =\left(\frac{1}{2^{p}}\right)^{l}\left(\Delta, d_{1}(Q)^{b_{1}} d_{2}(Q)^{b_{2}} \ldots d_{p}(Q)^{b_{p}} \bar{d}_{1}(Q)^{b_{1}} \bar{d}_{2}(Q)^{b_{2}} \ldots \bar{d}_{t}(Q)^{p_{p}}\right) \\
& =\left(\frac{1}{2^{p}}\right)^{l} \prod_{1 \leq i \leq p} \prod_{1 \leq c_{i} \leq b_{i}}(-2)^{c_{1}+2 c_{2}+\ldots+t c_{t}} B\left(c_{1}, c_{2}, \ldots, c_{t}\right)(\Delta, 1) \neq 0,
\end{aligned}
$$

where

$$
B\left(c_{1}, c_{2}, \ldots, c_{t}\right)=\prod_{i=1}^{t}\left(\sum_{s=i}^{t}\left(c_{s}+t-i\right)\right) .
$$

See [Zhu, pp. 106 and 116] for the last equality in the above computation.

Similarly for $l<0$, we have

$$
\begin{aligned}
& \left(\bar{\partial}_{p}(Q)^{-l} \Delta, d_{1}(Q)^{a_{1}} d_{2}(Q)^{a_{2}} \ldots d_{p}(Q)^{a_{p}} \bar{d}_{1}(Q)^{b_{1}} \bar{d}_{2}(Q)^{b_{2}} \ldots \bar{d}_{t}(Q)^{b_{p}}\right) \\
& =\left(\frac{1}{2^{p}}\right)^{-l} \prod_{1 \leq i \leq p} \prod_{1 \leq c_{i} \leq a_{i}}(-2)^{c_{1}+2 c_{2}+\ldots+t c_{t}} B\left(c_{1}, c_{2}, \ldots, c_{t}\right)(\Delta, 1) \neq 0 .
\end{aligned}
$$

We now examine the general (non-compact) case; i.e., $H=U(p, q)$.

Proposition 3.5. Assume that $p+q \leq n$. Let $\tau \in \widehat{\widetilde{K}}$ be such that

$$
\left.\tau^{*} \cong \rho^{\lambda} \otimes \rho{ }_{\text {q times }}^{\lambda^{*}+(\underbrace{-l, \ldots,-l}_{p \text { times }}, 0, \ldots, 0,-, l, \ldots,-l}\right)
$$

where $\lambda=\frac{p-q}{2} \mathbf{1}_{n}+\left(\alpha_{1}, \ldots, \alpha_{p}, 0, \ldots, 0,-\gamma_{q}, \ldots,-\gamma_{1}\right)$, and $\alpha_{i}, \gamma_{i}$ are integers satisfying

$$
\alpha_{1} \geq \ldots \geq \alpha_{p} \geq 0, \gamma_{1} \geq \ldots \geq \gamma_{q} \geq l, \quad \text { if } l>0,
$$

and

$$
\alpha_{1} \geq \ldots \geq \alpha_{p} \geq-l, \gamma_{1} \geq \ldots \geq \gamma_{q} \geq 0, \quad \text { if } l<0 .
$$

Then there exists a vector $\phi \in S_{\tau}$ such that $\langle\mathcal{D}, \phi\rangle \neq 0$. In particular, $\mathcal{D}$ has a non-zero $\sigma$-isotypic component for $\sigma \cong \tau^{*}$.

Since $\mathcal{D} \in\left(\Omega^{*}\right)^{p, q}(l)$, its $\sigma$-isotypic component $\mathcal{D}_{\sigma}$ clearly belongs to $\left(\Omega^{*}\right)^{p, q}(l)_{\sigma}$. The discussion on possible $\widetilde{K}$-types of $\left(\Omega^{*}\right)^{p, q}(l)$ before Proposition 3.4 together with Proposition 3.5 will therefore imply Theorem 3.3.

Proof of Proposition 3.5. We consider the following subgroups of $\operatorname{Sp}(4(p+q) n, \mathbb{R})$ :

$$
\begin{gathered}
L=H_{1} \times H_{2}=U(p) \times U(q), \\
E=E_{1} \times E_{2}=(U(p) \times U(p)) \times(U(q) \times U(q)), \\
N=N_{1} \times N_{2}=(U(n) \times U(n)) \times(U(n) \times U(n)),
\end{gathered}
$$

which fit into the following diamond dual pairs ([H2]): 


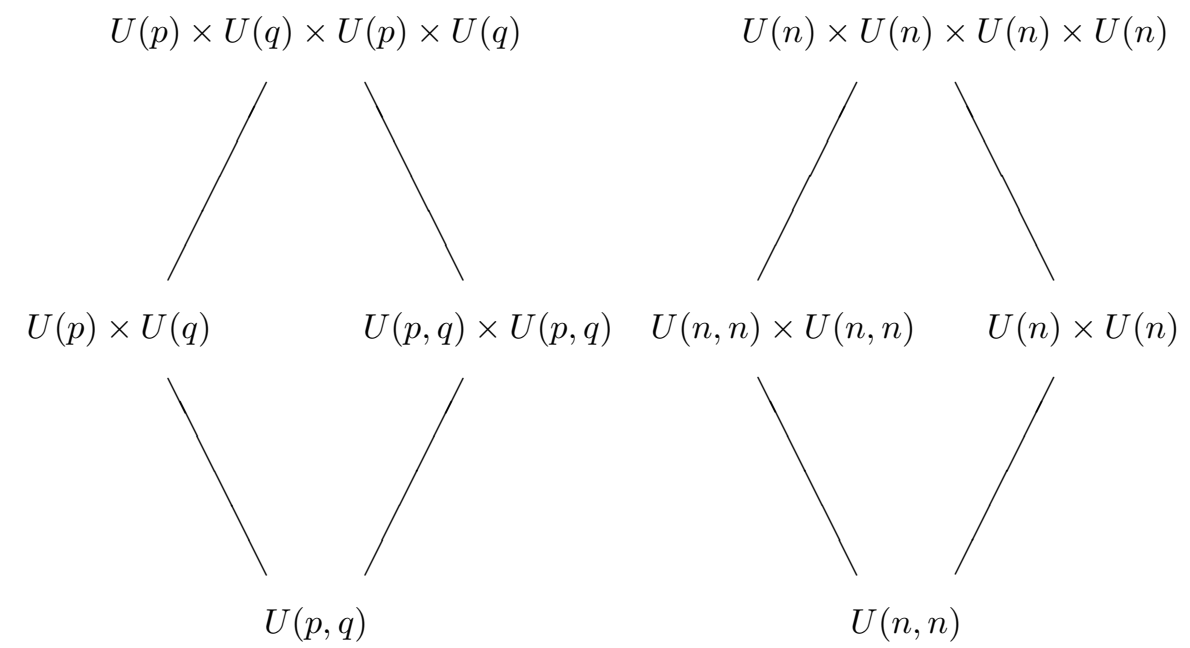

i.e. the pairs of Lie groups similarly placed in the two diamonds are reductive dual pairs. Let $\omega_{1}$ (resp. $\omega_{2}$ ) be the oscillator representation associated to the dual pair $\left(H_{1}, G\right)=(U(p), U(n, n)) \subseteq S p(4 p n, \mathbb{R})$ (resp. $\left(H_{2}, G\right)=(U(q), U(n, n) \subseteq$ $S p(4 q n, \mathbb{R})))$. Then we have the functorial property $([\mathrm{H} 5])$ :

$$
\left.\left.\left.\omega\right|_{\widetilde{E} \times \widetilde{N}} \cong \omega_{1}\right|_{\widetilde{E}_{1} \times \widetilde{N}_{1}} \otimes \omega_{2}\right|_{\widetilde{E}_{2} \times \widetilde{N}_{2}},
$$

where $\otimes$ here denotes outer tensor product. Let $Q=\left(q_{i j}\right), \bar{Q}=\left(\bar{q}_{i j}\right)(1 \leq i \leq p, 1 \leq$ $j \leq n)$ be the complex coordinates in the Fock model of $\omega_{1}$ and let $W=\left(w_{i j}\right)$, $\bar{W}=\left(\bar{w}_{i j}\right)(1 \leq i \leq q, 1 \leq j \leq n)$ be the complex coordinates in the Fock model of $\omega_{2}$. Let

$$
h_{1}(Q)=d_{1}(Q)^{a_{1}} d_{2}(Q)^{a_{2}} \ldots d_{p}(Q)^{a_{p}} \bar{d}_{1}(Q)^{b_{1}} \bar{d}_{2}(Q)^{b_{2}} \ldots \bar{d}_{p}(Q)^{b_{p}}
$$

be a highest weight vector under the action of $\widetilde{E}_{1} \times \widetilde{N}_{1}$. Similarly let

$$
h_{2}(W)=\widetilde{d}_{1}(W)^{c_{1}} \widetilde{d}_{2}(W)^{c_{2}} \ldots \widetilde{d}_{q}(W)^{c_{q}} \widetilde{\bar{d}}_{1}(W)^{e_{1}} \widetilde{\bar{d}}_{2}(W)^{e_{2}} \ldots \widetilde{\bar{d}}_{q}(W)^{e_{q}}
$$

be a highest weight vector under the action of $\widetilde{E}_{2} \times \widetilde{N}_{2}$, where

$$
d_{t}(Q)=\operatorname{det}\left(\begin{array}{ccc}
q_{11} & \ldots & q_{1 t} \\
\ldots & \ldots & \ldots \\
q_{t 1} & \ldots & q_{t t}
\end{array}\right), \quad 1 \leq t \leq \min (p, n),
$$

and

$$
\widetilde{d}_{t}(W)=\operatorname{det}\left(\begin{array}{ccc}
w_{1, n-t+1} & \ldots & w_{1, n} \\
\ldots & \ldots & \ldots \\
w_{t, n-t+1} & \ldots & w_{t, n}
\end{array}\right), \quad 1 \leq t \leq \min (q, n),
$$

and likewise for $\bar{d}_{t}(Q)$ and $\widetilde{\bar{d}}_{t}(W)$.

As usual, we denote the space of $K$-harmonics by $\mathcal{H}(K)$. For $\tau \in \widehat{\widetilde{K}}$, let $H(K)_{\tau}$ be the $\tau$-isotypic component of $\mathcal{H}(K)$. Then each $\mathcal{H}(K)_{\tau}$ is an irreducible $K^{\prime} \times \widetilde{K}$ module, where $K^{\prime}=U(p) \times U(q) \times U(p) \times U(q)([\mathrm{H} 2])$. Let $\tau \in \widehat{\widetilde{K}}$ be given as in the hypothesis. The results of Kashiwara and Vergne (Proposition 6.1 of [KV]) tells us that with appropriate choices of Borel subgroups, a $K^{\prime} \times \widetilde{K}$ joint highest 
weight vector $h$ in $\mathcal{H}(K)_{\tau}$ can be written as the product of two simultaneous highest weight vectors for the dual pairs $\left(E_{i}, N_{i}\right), i=1,2$; that is, $h\left(\begin{array}{c}Q \\ W\end{array}\right)=h_{1}(Q) h_{2}(W)$. Moreover the highest weight of $h_{1}$ (resp. $\left.h_{2}\right)$ is given precisely as in Proposition 3.4 (a) (resp., (b)).

We shall only consider the case $l>0$. The case of $l<0$ is similar. Note that $\mathcal{D}=\bar{\partial}_{p+q}^{l}(Z) \delta$ is mapped to the formal vector $\left(\frac{1}{\sqrt{2}}\right)^{(p+q) l} \partial_{p+q}^{l}\left(\begin{array}{c}Q \\ W\end{array}\right) \Delta$ in the Fock model (see eq. (3.5)). We claim that

$$
\left(\partial_{p+q}^{l}\left(\begin{array}{c}
Q \\
W
\end{array}\right) \Delta, h_{1}(Q) h_{2}(W)\right)=\left(\left(\begin{array}{cc}
\partial_{p}^{l}(Q) & 0 \\
0 & \partial_{q}^{l}(W)
\end{array}\right) \Delta, h_{1}(Q) h_{2}(W)\right) .
$$

The reason is as follows. We have $\frac{\partial}{\partial q_{i j}} \Delta=-\frac{1}{2} \bar{q}_{i j} \Delta$, and $\left(\bar{q}_{i j}\right)^{*}=2 \frac{\partial}{\partial \bar{q}_{i j}}$. Note that for $1 \leq i \leq p, p+1 \leq j \leq n, q_{i j}$ is an entry in the upper right corner of $\left(\begin{array}{c}Q \\ W\end{array}\right)$, and in this case, we have $\frac{\partial}{\partial \bar{q}_{i j}}\left(h_{1}(Q) h_{2}(W)\right)=0$.

Clearly we have $\Delta=\Delta_{1} \otimes \Delta_{2}$, where $\Delta_{i}$ is the image (in the Fock model of $\omega_{i}$ ) of the Dirac distribution at the origin of $V_{i}^{n}$, the direct sum of $n$-copies of the standard module for $H_{i}, i=1,2$, and $\otimes$ denotes the tensor product given in equation (3.6). If $h_{1}(Q)$ (resp. $h_{2}(W)$ ) is the highest weight vector in one of those $\widetilde{K}$-types given in Proposition 3.4 (a) (resp., (b)), then

$$
\left(\partial_{p+q}^{l}\left(\left(\begin{array}{c}
Q \\
W
\end{array}\right)\right) \Delta, h_{1}(Q) h_{2}(W)\right)=\left(\partial_{p}^{l}(Q) \Delta_{1}, h_{1}(Q)\right)\left(\partial_{q}^{l}(W) \Delta_{2}, h_{2}(W)\right) \neq 0,
$$

by our computations in Proposition 3.4.

Corollary 3.6. All the $\widetilde{K}$-types given in Propositions 2.1 and 2.2 occur in $\Omega^{p, q}(l)$ with multiplicity one.

\section{EMBEDding of $\Omega^{p, q}(l)$ INTO DEGENERATE PRINCIPAL SERIES}

In this section, we show that after twisting by a character of $\widetilde{U}(n, n)$, the Howe quotients $\Omega^{p, q}(0)$ for all $p$ and $q$ and $\Omega^{p, q}(l)(l \neq 0)$ for $p+q=n$ can be embedded into the degenerate series $I(s ; \nu)$ of $U(n, n)$ studied in [L] (see the introductory section for a description of $I(s ; \nu))$. Thus we can use the results in $[\mathrm{L}]$ to deduce the structure of these Howe quotients by identifying their images in the degenerate series.

Recall the reductive dual pair $(H, G)=(U(p, q), U(n, n)) \subseteq S p(4(p+q) n, \mathbb{R})$, and the action of $\widetilde{S} p(4(p+q) n, \mathbb{R})$ on $L^{2}\left(V^{n}\right)$ via the oscillator representation $\omega$. As before, we let $\mathcal{D}$ be the following tempered distribution on $V^{n}$ given by

$$
\mathcal{D}= \begin{cases}\delta, & \text { if } l=0, \\ \bar{\partial}_{p+q}^{l} \delta & \text { if } l>0, p+q \leq n, \\ \partial_{p+q}^{-l} \delta, & \text { if } l<0, p+q \leq n\end{cases}
$$

We have $\mathcal{D} \in\left(\Omega^{*}\right)^{p, q}(l) ;$ i.e, $h \cdot \mathcal{D}=(\operatorname{det} h)^{-l} \mathcal{D}$ for $h \in U(p, q)$.

Let $m=p+q$, as before. If $m$ is even, then $\widetilde{U}(n, n)$ splits over $U(n, n)$, and if $m$ is odd, then $\widetilde{U}(n, n)$ is isomorphic to the cover defined by the character det ${ }^{\frac{m}{2}}$ of $\widetilde{U}(n, n)([\mathrm{Ad}])$. The image of the covering group $\widetilde{M}$ in $\widetilde{U}(n, n)$ can then be identified as a set with $\left\{\left(m_{a}, \lambda\right): a \in G L(n, \mathbb{C}), \lambda= \pm\left(\frac{\operatorname{det} a}{|\operatorname{det} a|}\right)^{m}\right\}$. 
Let $\xi$ be the following character of $\widetilde{U}(n, n)$ :

$$
\xi= \begin{cases}\text { trivial, } & \text { if } m \text { is even, } \\ \operatorname{det}^{\frac{m}{2}}, & \text { if } m \text { is odd }\end{cases}
$$

We have $\xi\left(\left(m_{a}, \lambda\right)\right)=\lambda$, for $\left(m_{a}, \lambda\right) \in \widetilde{M}$. Let

$$
\omega_{\xi}=\xi^{-1} \otimes \omega, \quad \Omega_{\xi}^{p, q}(l)=\xi^{-1} \otimes \Omega^{p, q}(l) .
$$

Then $\omega_{\xi}$ factors through $U(n, n)$, and $\Omega_{\xi}^{p, q}(l)$ is a $U(n, n)$ module.

We shall identify the maximal parabolic subgroup $P=M N$ with the subgroup of $U(n, n)$ fixing $V^{n} \cong M_{p+q, n}(\mathbb{C})$. Recall that the modular function of $P$ is given by

$$
\Delta(p)=\Delta\left(m_{a} n_{b}\right)=|\operatorname{det} a|^{2 n}, \quad p=m_{a} n_{b} \in P .
$$

Now we have

$$
\begin{aligned}
{\left[\omega\left(\left(m_{a}, \lambda\right)\right) f\right](x) } & =|\operatorname{det} a|^{m} f(x a), \\
{\left[\omega\left(n_{b}\right) f\right](x) } & =e^{\frac{1}{2} \operatorname{tr}\left(I_{p, q} x b x^{*}\right)} f(x),
\end{aligned}
$$

for $\left(m_{a}, \lambda\right) \in \widetilde{M}, n_{b} \in N, \quad f \in L^{2}\left(M_{p+q, n}(\mathbb{C})\right), x \in M_{p+q, n}(\mathbb{C})$. Thus

$$
\left[\omega_{\xi}\left(m_{a}\right) f\right](x)= \begin{cases}\chi_{m ; 0}\left(m_{a}\right) f(x a), & \text { if } m \text { is even, } \\ \chi_{m,-m}\left(m_{a}\right) f(x a), & \text { if } m \text { is odd, }\end{cases}
$$

and therefore

$$
\omega_{\xi}(y) \cdot \delta= \begin{cases}\chi_{m, 0}^{-1}(y) \delta, & \text { if } m \text { is even, } \\ \chi_{m,-m}^{-1}(y) \delta, & \text { if } m \text { is odd }\end{cases}
$$

where $y=m_{a} n_{b} \in P$ and we extend the characters of $M$ to $P$ by letting $N$ act trivially.

For any $\phi \in \mathcal{S}\left(V^{n}\right)$, we consider the function

$$
\mathcal{D}_{\phi}(g)=\mathcal{D}\left(\omega_{\xi}(g) \phi\right), \quad g \in G=U(n, n) .
$$

For $l=0$, we compute

$$
\begin{gathered}
\mathcal{D}_{\phi}\left(n_{b} m_{a} g\right)=\delta\left(\omega_{\xi}\left(n_{b} m_{a} g\right) \phi\right)=\left(\omega_{\xi}\left(m_{a}^{-1} n_{b}^{-1}\right) \cdot \delta\right)\left(\omega_{\xi}(g) \phi\right) \\
= \begin{cases}\chi_{m, 0}\left(n_{b} m_{a}\right) \mathcal{D}_{\phi}(g)=\chi_{m-n, 0}\left(n_{b} m_{a}\right) \Delta\left(n_{b} m_{a}\right)^{\frac{1}{2}} \mathcal{D}_{\phi}(g), & \text { if } m \text { is even, } \\
\chi_{m,-m}\left(n_{b} m_{a}\right) \mathcal{D}_{\phi}(g)=\chi_{m-n,-m}\left(n_{b} m_{a}\right) \Delta\left(n_{b} m_{a}\right)^{\frac{1}{2}} \mathcal{D}_{\phi}(g), & \text { if } m \text { is odd, }\end{cases}
\end{gathered}
$$

where $\Delta$ is the modular function of $P$. Thus the function $\mathcal{D}_{\phi}$ is in the space of the induced representation $I(m-n ; 0)$ or $I(m-n ;-m)$ depending on whether $m$ is even or odd. Consequently we obtain a map

$$
\lambda: \phi \longrightarrow \mathcal{D}_{\phi}
$$

from $\mathcal{S}\left(V^{n}\right)$ to certain induced representation $I(s ; \nu)$. We now restrict the map to the space $S \subseteq \mathcal{S}\left(V^{n}\right)$. Because of the way Howe's quotient $\Omega_{\xi}^{p, q}$ is defined and the transformation property of $\mathcal{D}$, this restriction map factors through $\Omega_{\xi}^{p, q}$. To summarize, we have

$$
\lambda: \Omega_{\xi}^{p, q} \longrightarrow I(s ; \nu)= \begin{cases}I(m-n ; 0), & \text { if } m \text { is even } \\ I(m-n ;-m), & \text { if } m \text { is odd }\end{cases}
$$


For $l \neq 0$, if we further assume that $m=n$, then a similar computation shows that for $n$ even,

$$
\lambda: \Omega_{\xi}^{p, q}(l) \longrightarrow I(s ; \nu)=I(|l| ;-l),
$$

and for $n$ odd,

$$
\lambda: \Omega_{\xi}^{p, q}(l) \longrightarrow I(s ; \nu)=I(|l| ;-n-l) .
$$

Theorem 4.1. Assume either (i) $l=0$, or (ii) $l \neq 0$ and $m=n$. Then we have $a$ G-equivariant embedding:

$$
\lambda: \Omega_{\xi}^{p, q}(l) \hookrightarrow I(s ; \nu) .
$$

Here $s$ and $\nu$ are as given in equations (4.2), (4.3) and (4.4).

Proof. $\lambda$ is easily checked to be $G$-equivariant. We only need to show injectivity. We fix an element $\phi$ of $S$ with nonzero image $\bar{\phi}$ in the quotient $\Omega_{\xi}^{p, q}(l)$. We may assume that some isotypic component of $\bar{\phi}$, denoted by $\bar{\phi}_{\tau_{\xi}}$, is not zero, where $\tau_{\xi}=\xi^{-1} \otimes \tau$, and $\tau$ is one of the $\widetilde{K}$-types given in Propositions 2.1 and 2.2.

Suppose that $\lambda(\bar{\phi})=0$, then since $\lambda$ is $G$-equivariant, we have $\lambda\left(\bar{\phi}_{\tau_{\xi}}\right)=0$. We replace $\omega$ by $\omega_{\xi}$ in the definition of $\left(\Omega^{*}\right)^{p, q}(l)$ to get $\left(\Omega^{*}\right)_{\xi}^{p, q}(l)$, and let $\mathcal{D}_{\tau_{\xi}^{*}} \in$ $\left(\Omega^{*}\right)_{\xi}^{p, q}(l)_{\tau_{\xi}^{*}}$ be the corresponding $\tau_{\xi}^{*}$-isotypic component of $\mathcal{D}$, which we know to be non-zero. For any $k \in K$, we have

$$
0=\lambda\left(\bar{\phi}_{\tau_{\xi}}\right)(k)=\mathcal{D}\left(\omega_{\xi}(k)\right) \bar{\phi}_{\tau_{\xi}}=\mathcal{D}_{\tau_{\xi}^{*}}\left(\omega_{\xi}(k) \bar{\phi}_{\tau_{\xi}}\right)=\left\langle\mathcal{D}_{\tau_{\xi}^{*}}, \omega_{\xi}(k) \bar{\phi}_{\tau_{\xi}}\right\rangle .
$$

Since $\Omega_{\xi}^{p, q}(l)_{\tau_{\xi}}$ is multiplicity one, $\left\{\omega_{\xi}(k) \bar{\phi}_{\tau_{\xi}} \mid k \in K\right\}$ spans $\Omega_{\xi}^{p, q}(l)_{\tau_{\xi}}$. Since $0 \neq$ $\mathcal{D}_{\tau_{\xi}^{*}} \in\left(\Omega^{*}\right)_{\xi}^{p, q}(l)_{\tau_{\xi}^{*}}$, and since the pairing between $\Omega^{p, q}(l)$ and $\left(\Omega^{*}\right)^{p, q}(l)$ is nondegenerate (see the discussion before Proposition 3.4), we see that the last expression cannot be identically zero. We have a contradiction. This proves that $\lambda: \Omega_{\xi}^{p, q}(l) \rightarrow I(s ; \nu)$ is injective.

\section{Identification of COnstituents through LOCAL THETA CORRESPONDENCE}

In this section, we shall describe the image of $\Omega_{\xi}^{p, q}(l)$ under the embedding $\lambda: \Omega_{\xi}^{p, q}(l) \hookrightarrow I(s ; \nu)$ given in Theorem 4.1. Since $I(s ; \nu)$ is multiplicity free as a $K$-module, the image of $\Omega_{\xi}^{p, q}(l)$ in $I(s ; \nu)$ is completely determined by the $K$ types in $\Omega_{\xi}^{p, q}(l)$. Since the detailed structure of $I(s ; \nu)$ is described in [L], we can deduce the reducibility, composition series and unitarity of $\Omega_{\xi}^{p, q}(l)$. On the other hand, for different values of $p$ and $q, \Omega_{\xi}^{p, q}(l)$ is embedded into the same induced representation $I(s ; \nu)$, provided that $p+q$ is fixed. We shall show that in the case $l=0$ and $p+q \geq n$, every constituent in $I(p+q-n ; 0)$ with $p+q$ even and in $I(p+q-n ;-p-q)$ with $p+q$ odd is isomorphic to a quotient of submodules which are intersections of the images of some of the $\Omega_{\xi}^{p, q}$, in a very precise way.

We shall now summarize some results in $[\mathrm{L}]$. Recall that the induced representation $I(s ; \nu)$ can be identified with a function space $S^{\alpha, \beta}\left(X^{o o}\right)$, where $\alpha=-\frac{s+n-\nu}{2}$ and $\beta=-\frac{s+n+\nu}{2}$. We shall now describe this space. Let $U(n, n)$ act on the space $M_{2 n, n}(\mathbb{C})$ of $2 n \times n$ complex matrices by

$$
g \cdot x=\left(g^{-1}\right)^{t} x, \quad g \in U(n, n), x \in M_{2 n, n}(\mathbb{C}),
$$


and let $X^{o o}$ be the $U(n, n)$ orbit of $x_{o}=\left(\begin{array}{c}I_{n} \\ -I_{n}\end{array}\right)$. Here $g^{t}$ denotes the transpose of $g$ and $I_{n}$ denotes the $n \times n$ identity matrix. For $\alpha, \beta \in \mathbb{C}$ such that $\alpha-\beta \in \mathbb{Z}$, let

$$
\begin{aligned}
S^{\alpha, \beta}\left(X^{o o}\right)= & \left\{f \in C^{\infty}\left(X^{o o}\right):\right. \\
& \left.\quad f(x a)=(\operatorname{det} a)^{\alpha}(\overline{\operatorname{det} a})^{\beta} f(x), \forall x \in X^{o o}, a \in G L(n, \mathbb{C})\right\},
\end{aligned}
$$

and let $U(n, n)$ act on it by

$$
(g . f)(x)=f\left(g^{t} x\right), \quad g \in U(n, n), x \in X^{o o} .
$$

$S^{\alpha, \beta}\left(X^{o o}\right)$ admits the following decomposition into a sum of $K$-types:

$$
S^{\alpha, \beta}\left(X^{o o}\right)=\sum_{\lambda \in \Lambda_{n}^{+}} V_{\lambda}
$$

where $V_{\lambda}$ is isomorphic to $\rho^{\lambda} \otimes \rho^{\lambda^{*}+(\alpha-\beta) \mathbf{1}_{n}}$ as a representation of $K$. For $1 \leq j \leq n$, let

$$
e_{j}=(\underbrace{0, \ldots, 0,1}_{j}, 0, \ldots, 0) \in \mathbb{Z}^{n} .
$$

It is proved in [L] (see Corollary 6.1 of [L]) that a $K$-type $V_{\lambda}$ can be transformed to the $K$-type $V_{\lambda+e_{j}}$ (respectively $\left.V_{\lambda-e_{j}}\right)(1 \leq j \leq n)$ if and only if the transition coefficient $\alpha-\lambda_{j}+j-1$ (respectively $\beta+\lambda_{j}+n-j$ ) is nonzero. Thus $S^{\alpha, \beta}\left(X^{o o}\right)$ is irreducible if and only if both $\alpha, \beta \notin \mathbb{Z}$. In the case when $S^{\alpha, \beta}\left(X^{o o}\right)$ is reducible, i.e., when both $\alpha, \beta \in \mathbb{Z}$, the hyperplanes

$$
\ell_{j}^{+}: \lambda_{j}=\alpha+j-1, \quad \ell_{j}^{-}: \lambda_{j}=-(\beta+n-j), \quad(1 \leq j \leq n)
$$

divide $S^{\alpha, \beta}\left(X^{o o}\right)$ into a number of irreducible constituents. Each of these constituents is a subquotient of $S^{\alpha, \beta}\left(X^{o o}\right)$. We can then construct a graph which captures the most important information on the module structure of $S^{\alpha, \beta}\left(X^{o o}\right)$. This graph is called the module diagram of $S^{\alpha, \beta}\left(X^{o o}\right)$. The nodes of this graph are the irreducible constituents of $S^{\alpha, \beta}\left(X^{o o}\right)$, and if there is an edge joining two constituents $R_{1}$ and $R_{2}$ with $R_{1}$ placed at a higher position, then the vectors in $R_{1}$ can be transformed to the vectors in $R_{2}$. The readers are advised to refer either to $[\mathrm{Al}]$ or section 7 of [L] for a more precise definition of module diagram. It turns out that the module diagram for $S^{\alpha, \beta}\left(X^{o o}\right)$ is a (complete or incomplete) triangle, and its pattern depends on the number $i=\alpha+\beta+n-1$. The module diagrams of all the $S^{\alpha, \beta}\left(X^{o o}\right)$ are also given in $[\mathrm{L}]$.

We shall first study $\Omega_{\xi}^{p, q}=\Omega_{\xi}^{p, q}(0)$. We let $m$ be a positive integer and consider all pairs of nonnegative integers $(p, q)$ such that $p+q=m$. Then by Theorem 4.1, we have $U(n, n)$ embeddings

$$
\lambda: \Omega_{\xi}^{p, q} \hookrightarrow \begin{cases}I(m-n ; 0) \cong S^{-\frac{m}{2},-\frac{m}{2}}\left(X^{o o}\right), & m \text { even } \\ I(m-n ;-m) \cong S^{-m, 0}\left(X^{o o}\right), & m \text { odd }\end{cases}
$$

Recall that the pattern of the module diagram of $S^{\alpha, \beta}\left(X^{o o}\right)$ depends on the number (see section 7 of $[\mathrm{L}]) i=\alpha+\beta+n-1$ and its sign. Let

$$
(\alpha, \beta)= \begin{cases}\left(-\frac{m}{2},-\frac{m}{2}\right), & \text { if } m \text { is even } \\ (-m, 0), & \text { if } m \text { is odd }\end{cases}
$$

Then we have $i=-m+n-1$. Hence we shall consider 2 cases separately, i.e., $1 \leq m \leq n-1$ and $m \geq n$. 


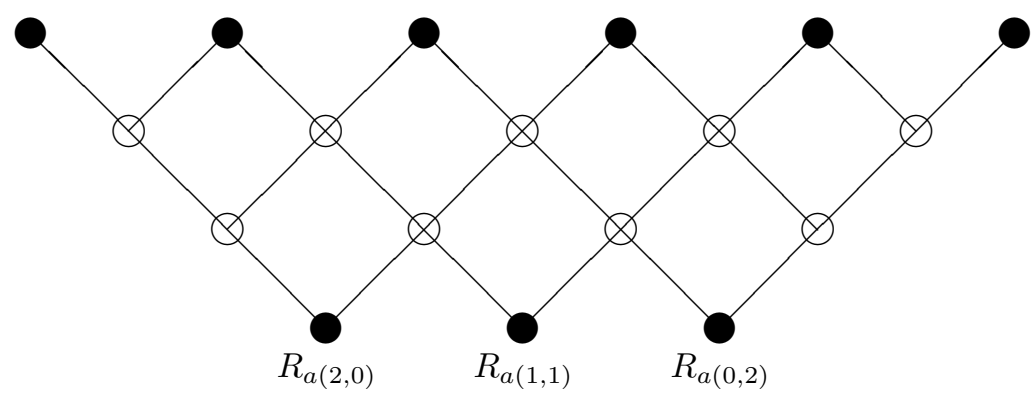

Figure 1

Case 1. $1 \leq m \leq n-1$. In this case, we have $0 \leq i \leq n-2$. We shall first recall a description of the irreducible constituents in $S^{\alpha, \beta}\left(X^{o o}\right)$ when $0 \leq i=\alpha+\beta+n-1 \leq$ $n-2$, as given in [L].

The hyperplanes $\ell_{j}^{ \pm}(1 \leq j \leq n)$ divide $\Lambda_{n}^{+}$into the following disjoint subsets:

$$
\begin{aligned}
& A_{1}^{j}=\left\{\lambda \in \Lambda_{n}^{+}: \lambda_{j}<-(\beta+n-j),\right. \\
& A_{2}^{j}=\left\{\lambda \in \Lambda_{n}^{+}:-(\beta+n-j) \leq \lambda_{j} \leq \alpha+j-1\right\}, \\
& A_{3}^{j}=\left\{\lambda \in \Lambda_{n}^{+}: \lambda_{j}>\alpha+j-1\right\} .
\end{aligned}
$$

For $0 \leq s, t \leq n$ with $s+t \leq n$, let $R_{a(s, t)}$ be the direct sum of all the $K$-types $V_{\lambda}$ with $\lambda$ in the set

$$
\left(A_{3}^{1} \cap \cdots \cap A_{3}^{s}\right) \cap\left(A_{2}^{s+1} \cap \cdots \cap A_{2}^{n-t}\right) \cap\left(A_{1}^{n-t+1} \cap \cdots \cap A_{1}^{n}\right) .
$$

If $R_{a(s, t)}$ is nonempty, then it forms an irreducible constituent in $S^{\alpha, \beta}\left(X^{o o}\right)$. We shall frequently abuse notation and identify $R_{a(s, t)}$ with the subset of $\Lambda_{n}^{+}$given in eq. (5.1). We shall do the same for all other irreducible constituents defined later. The module diagram of $S^{\alpha, \beta}\left(X^{o o}\right)$ is a "incomplete inverted triangle" with $i+2$ rows (i.e. the lowest $n-i-1$ rows in the complete triangle has been removed). For example, the module diagram for $S^{-\frac{m}{2},-\frac{m}{2}}\left(X^{o o}\right)$ in the case when $n=5$ and $m=2$ is given in Fig. 1. Here a blacken circle represents a unitary constituent.

For $S^{-\frac{m}{2},-\frac{m}{2}}\left(X^{o o}\right)$ and $S^{-m, 0}\left(X^{o o}\right)$, the constituents at the lowest row of the diagram are

$$
R_{a(m, 0)}, \quad R_{a(m-1,1)}, \quad R_{a(m-2,2)}, \quad \ldots, R_{a(0, m)} .
$$

By Theorem 9.18 of [L], each of these constituents is an irreducible unitary submodule of $S^{-\frac{m}{2},-\frac{m}{2}}\left(X^{o o}\right)$ or $S^{-m, 0}\left(X^{o o}\right)$.

Proposition 5.1. If $p+q=m$ and $m$ is an integer such that $1 \leq m \leq n-1$, then

$$
\lambda\left(\Omega_{\xi}^{p, q}\right)=R_{a(p, q)} \subseteq \begin{cases}I(m-n ; 0), & \text { if } m \text { is even }, \\ I(m-n ;-m), & \text { if } m \text { is odd. }\end{cases}
$$

Hence $\Omega^{p, q}$ is irreducible and unitary. 
Proof. We first assume that $m$ is even. By Proposition 2.1 and Corollary 3.6, the $K$-types in $\Omega_{\xi}^{p, q}$ are exactly those of the form $\rho^{\lambda} \otimes \rho^{\lambda^{*}}$ and

$$
\lambda=\frac{p-q}{2} \mathbf{1}_{n}+\left(\alpha_{1}, \ldots, \alpha_{p}, 0, \ldots, 0,-\gamma_{q}, \ldots,-\gamma_{1}\right)
$$

where $\alpha_{1} \geq \alpha_{2} \geq \cdots \geq \alpha_{p} \geq 0$ and $\gamma_{1} \geq \gamma_{2} \geq \cdots \geq \gamma_{q} \geq 0$. On the other hand the $K$-types in $R_{a(p, q)} \subseteq S^{-\frac{m}{2},-\frac{m}{2}}\left(X^{o o}\right)$ are of the form $\rho^{\lambda} \otimes \rho^{\lambda^{*}}$ where $\lambda$ satisfies

(i) $\lambda_{j} \geq \alpha+j, \quad 1 \leq j \leq p$,

(ii) $-(\beta+n-j) \leq \lambda_{j} \leq \alpha+j-1, \quad p+1 \leq j \leq n-q$,

(iii) $\lambda_{j} \leq-(\beta+n-j)-1, \quad n-q+1 \leq j \leq n$,

where $\alpha=\beta=-\frac{m}{2}$. Since $\lambda_{1} \geq \lambda_{2} \geq \cdots \geq \lambda_{n}$, (i)-(iii) are equivalent to

(i)' $\lambda_{j} \geq \alpha+p, \quad 1 \leq j \leq p$.

(ii)' $-(\beta+q) \leq \lambda_{j} \leq \alpha+p, \quad p+1 \leq j \leq n-q$,

(iii)' $\lambda_{j} \leq-(\beta+q), \quad n-q+1 \leq j \leq n$.

Observe since $\alpha=\beta=-\frac{p+q}{2}, \alpha+p=-(\beta+q)=\frac{p-q}{2}$. Thus (i)'-(iii)' are equivalent to (5.2). Hence $\Omega_{\xi}^{p, q}$ and $R_{a(p, q)}$ have the same collection of $K$-types.

Next we assume that $m$ is odd. In this case the $K$-types in $\Omega_{\xi}^{p, q}$ are exactly those of the form $\rho^{\lambda-\frac{m}{2} \mathbf{1}_{n}} \otimes \rho^{\lambda^{*}-\frac{m}{2}} \mathbf{1}_{n}$ where $\lambda$ satisfies the condition (5.2). If we let $\mu=\lambda-\frac{m}{2} \mathbf{1}_{n}$, then $\rho^{\lambda-\frac{m}{2} \mathbf{1}_{n}} \otimes \rho^{\lambda^{*}-\frac{m}{2} \mathbf{1}_{n}}=\rho^{\mu} \otimes \rho^{\mu^{*}-m \mathbf{1}}$ and

$$
\mu=-q+\left(\alpha_{1}, \ldots, \alpha_{p}, 0, \ldots, 0-\gamma_{q}, \ldots,-\gamma_{1}\right)
$$

where $\alpha_{1} \geq \alpha_{2} \geq \cdots \geq \alpha_{p} \geq 0$ and $\gamma_{1} \geq \gamma_{2} \geq \cdots \geq \gamma_{q} \geq 0$. It can be checked in a similar way that these are exactly the $K$-types which occur in $R_{a(p, q)} \subseteq$ $S^{-m, 0}\left(X^{o o}\right)$.

We shall denote the unique irreducible quotient of $\Omega_{\xi}^{p, q}$ by $Q_{\xi}^{p, q}$. Then by the above proposition, if $1 \leq p+q \leq n-1$, then $Q_{\xi}^{p, q}=\Omega_{\xi}^{p, q}$. In particular $Q_{\xi}^{p, q}$ is unitary.

Case 2. $m \geq n$. In this case, we have $i \leq-1$ where $i=\alpha+\beta+n-1$. The hyperplanes $\ell_{j}^{ \pm}(1 \leq j \leq n)$ divide $\Lambda_{n}^{+}$into the following subsets:

$$
\begin{aligned}
& B_{1}^{j}=\left\{\lambda \in \Lambda_{n}^{+}: \lambda_{j} \leq \alpha+j-1\right\}, \\
& B_{2}^{j}=\left\{\lambda \in \Lambda_{n}^{+}: \alpha+j \leq \lambda_{j} \leq-(\beta+n-j)-1\right\}, \\
& B_{3}^{j}=\left\{\lambda \in \Lambda_{n}^{+}: \lambda_{j} \geq-(\beta+n-j)\right\} .
\end{aligned}
$$

Again for $0 \leq s, t \leq n$ with $s+t \leq n$, we let $R_{a(s, t)}$ be the direct sum of all the $K$-types $V_{\lambda}$ with $\lambda$ in the set

$$
\left(B_{3}^{1} \cap \cdots \cap B_{3}^{s}\right) \cap\left(B_{2}^{s+1} \cap \cdots \cap B_{2}^{n-t}\right) \cap\left(B^{n-t+1} \cap \cdots \cap B_{1}^{n}\right),
$$

If this set is nonempty, then $R_{a(s, t)}$ forms an irreducible constituent of $S^{\alpha, \beta}\left(X^{o o}\right)$

We now assume that $m=n$, so we have

$$
(\alpha, \beta)= \begin{cases}\left(-\frac{n}{2},-\frac{n}{2}\right) & \text { if } n \text { is even, } \\ (-n, 0) & \text { if } n \text { is odd. }\end{cases}
$$

Then $S^{\alpha, \beta}\left(X^{o o}\right)$ is on the unitary axis (see Proposition 9.1 of $[\mathrm{L}]$ ) and

$$
S^{\alpha, \beta}\left(X^{o o}\right)=\bigoplus_{j=0}^{n} R_{a(j, n-j)}
$$


is a decomposition of $S^{\alpha, \beta}\left(X^{o o}\right)$ into a direct sum of $n+1$ irreducible unitary submodules. Hence the module diagram of $S^{\alpha, \beta}\left(X^{o o}\right)$ consist of only one row of $n+1$ nodes with no edge.

If $n$ is even, then by Proposition 2.1 and Corollary 3.6, the $K$-types of $\Omega_{\xi}^{p, q}$ are exactly those of the form $\rho^{\lambda} \otimes \rho^{\lambda^{*}}$ where

$$
\lambda=\frac{p-q}{2} \mathbf{1}_{n}+\left(\alpha_{1}, \alpha_{2}, \ldots, \alpha_{p},-\gamma_{q}, \ldots,-\gamma_{1}\right)
$$

and $\alpha_{1} \geq \alpha_{2} \geq \cdots \geq \alpha_{p} \geq 0$ and $\gamma_{1} \geq \gamma_{2} \geq \cdots \geq \gamma_{q} \geq 0$. Now for $\alpha=\beta=-\frac{n}{2}$, we have $\frac{p-q}{2}=-(\beta+n-p)=\alpha+(p+1)-1$. Hence $\rho^{\lambda} \otimes \rho^{\lambda^{*}}$ occurs in $\Omega_{\xi}^{p, q}$ if and only if $\lambda_{p} \geq-(\beta+n-p)$ and $\lambda_{p+1} \leq \alpha+(p+1)-1$; that is $\lambda \in R_{a(p, q)} \subseteq S^{-\frac{n}{2},-\frac{n}{2}}\left(X^{o o}\right)$. Consequently $\lambda\left(\Omega_{\xi}^{p, q}\right)=R_{a(p, q)} \subseteq S^{-\frac{n}{2},-\frac{n}{2}}\left(X^{o o}\right)$.

If $n$ is odd, then the $K$-types of $\Omega_{\xi}^{p, q}$ are exactly those of the form $\rho^{\lambda} \otimes \rho^{\lambda^{*}-m \mathbf{1}}$ where

$$
\lambda=-q+\left(\alpha_{1}, \alpha_{2}, \ldots, \alpha_{p},-\gamma_{q}, \ldots,-\gamma_{1}\right) .
$$

For $\alpha=-n$ and $\beta=0$, we have $\alpha+(p+1)-1=-(\beta+n-p)=-q$. Thus as before, $\lambda \geq-(\beta+n-p)$ and $\lambda_{p+1} \leq \alpha+(p+1)-1$ which shows that $\lambda \in R_{a(p, q)} \subseteq$ $S^{-n, 0}\left(X^{o o}\right)$. Hence we obtain the following proposition.

Proposition 5.2. Assume that $m=n$. Let

$$
(s, \nu)= \begin{cases}(0,0) & \text { if } n \text { is even }, \\ (0,-n) & \text { if } n \text { is odd }\end{cases}
$$

Then

$$
\lambda\left(\Omega_{\xi}^{p, q}\right)=R_{a(p, q)} \subseteq I(s ; \nu) .
$$

Hence $\Omega_{\xi}^{p, q}$ is irreducible and unitary. Moreover,

$$
I(s ; \nu)=\bigoplus_{p+q=n} \lambda\left(\Omega_{\xi}^{p, q}\right) .
$$

It again follows that in this case $Q_{\xi}^{p, q}=\Omega_{\xi}^{p, q}$ and that $Q_{\xi}^{p, q}$ is unitary.

We remark that the fact $Q_{\xi}^{p, q}$ is unitary when $p+q \leq n$ also follows from general results on theta liftings for stable dual pairs $([\mathrm{Li}])$.

Corollary 5.3. Let $1 \leq m \leq n$.

(i) If $m$ is even, then every irreducible unitary submodule of $I(m-n ; 0)$ is the image of some $\Omega_{\xi}^{p, q}$ where $p+q=m$.

(ii) If $m$ is odd, then every irreducible unitary submodule of $I(m-n ;-m)$ is the image of some $\Omega_{\xi}^{p, q}$ where $p+q=m$.

Next we assume that $m \geq n+1$. Since in this case, we have $i=-m+n-1 \leq-2$, the module diagram for $S^{-\frac{m}{2},-\frac{m}{2}}\left(X^{o o}\right)$ or $S^{-m, 0}\left(X^{o o}\right)$ is a (upright) complete or incomplete triangle. If $m \geq 2 n$ (so that $i \leq-(n+1)$ ), then the module diagram for $S^{-\frac{m}{2},-\frac{m}{2}}\left(X^{o o}\right)$ or $S^{-m, 0}\left(X^{o o}\right)$ is a complete triangle with $n+1$ rows (see Fig. 2 ). If $n+1 \leq m \leq 2 n-1$, then $-n \leq i \leq-2$ and the diagram for $S^{-\frac{m}{2},-\frac{m}{2}}\left(X^{o o}\right)$ or $S^{-m, 0}\left(X^{o o}\right)$ consists of only the lowest $|i|$ rows of the complete triangle.

If $R_{a\left(d_{1}, d_{2}\right)}$ is an irreducible constituent in $S^{-\frac{m}{2},-\frac{m}{2}}\left(X^{o o}\right)$ (resp. $S^{-m, 0}\left(X^{o o}\right)$ ), we let $M\left(d_{1}, d_{2}\right)$ be the submodule of $S^{-\frac{m}{2},-\frac{m}{2}}\left(X^{o o}\right)$ (resp. $\left.S^{-m, 0}\left(X^{o o}\right)\right)$ generated 


$$
R_{a(0,0)}
$$

$$
R_{a(1,0)} \quad R_{a(0,1)}
$$

$R_{a(2,0)}$

$R_{a(1,1)}$

$R_{a(0,2)}$

$R_{a(n, 0)}$

$R_{a(n-1,1)}$

$R_{a(1, n-1)}$

$R_{a(0, n)}$

FiguRE 2

by $R_{a\left(d_{1}, d_{2}\right)}$. Specifically,

$$
M\left(d_{1}, d_{2}\right)=\bigoplus\left\{R_{a(s, t)}: s \geq d_{1}, t \geq d_{2}\right\} .
$$

Note that the module diagram for $M\left(d_{1}, d_{2}\right)$ is a "subtriangle" in the module diagram for $S^{-\frac{m}{2},-\frac{m}{2}}\left(X^{o o}\right)$ (resp. $S^{-m, 0}\left(X^{o o}\right)$ (see Fig. 3).

Proposition 5.4. Let $m$ be integer such that $m \geq n+1$ and let $p+q=m$.

(i) We have

$$
\lambda\left(\Omega_{\xi}^{p, q}\right)=M\left(d_{1}, d_{2}\right)
$$

and

$$
Q_{\xi}^{p, q} \cong R_{a\left(d_{1}, d_{2}\right)},
$$

where $d_{1}=\max \{0, n-q\}$ and $d_{2}=\max \{0, n-p\}$.

(ii) If $n+1 \leq m \leq 2 n$, then $Q_{\xi}^{p, q}$ is unitary if and only if $p \leq n$ and $q \leq n$ or $p q=0$.

(iii) If $m \geq 2 n+1$, then $Q_{\xi}^{p, q}$ is unitary if and only if $p q=0$.

Proof. We shall only prove the case when $m$ is even. We first consider (i). Assume that $n+1 \leq m \leq 2 n$. In this case we observe that a $K$-type $\rho^{\lambda} \otimes \rho^{\lambda^{*}}$ occurs in $\Omega_{\xi}^{p, q}$ if and only if $\lambda$ is of the form

$$
\begin{aligned}
& \lambda=\left\{\begin{array}{cc}
\frac{p-q}{2} \mathbf{1}_{n}+\left(\alpha_{1}-\gamma_{n}, \ldots, \alpha_{p}-\gamma_{n-p+1},-\gamma_{n-p}, \ldots,-\gamma_{1}\right), & \text { if } 0 \leq p \leq m-n, \\
\frac{p-q}{2} \mathbf{1}_{n}+\left(\alpha_{1}, \ldots, \alpha_{n-q}, \alpha_{n-q+1}-\gamma_{q}, \ldots, \alpha_{p}-\gamma_{n-p+1},\right. \\
\left.-\gamma_{n-p}, \ldots,-\gamma_{1}\right), & \text { if } m-n+1 \leq p \leq n-1, \\
\frac{p-q}{2} \mathbf{1}_{n}+\left(\alpha_{1}, \ldots, \alpha_{n-q}, \alpha_{n-q+1}-\gamma_{q}, \ldots, \alpha_{n}-\gamma_{1}\right), & \text { if } n \leq p \leq m,
\end{array}\right.
\end{aligned}
$$

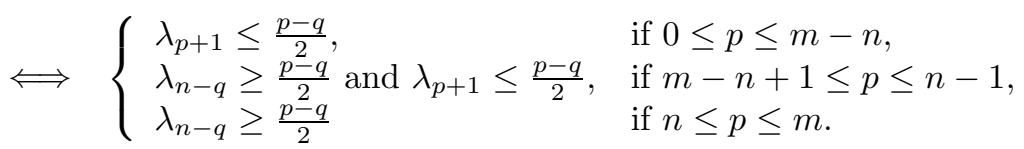




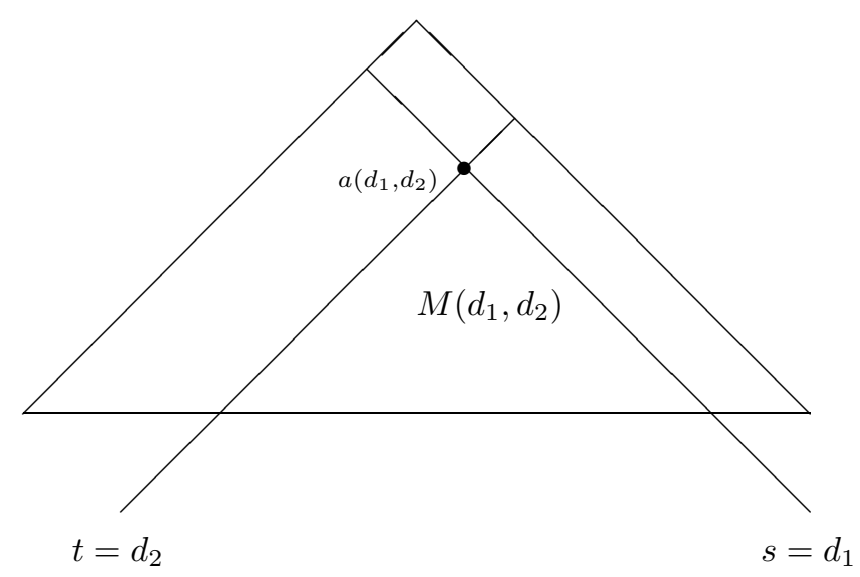

$m \geq 2 n$

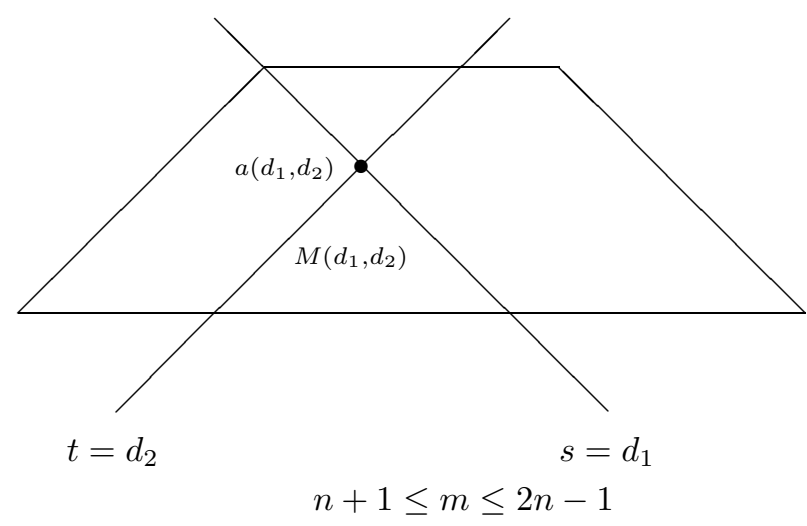

Figure 3

Consequently,

$$
\begin{aligned}
\lambda\left(\Omega_{\xi}^{p, q}\right)= & \begin{cases}B_{1}^{p+1}=\bigoplus_{t \geq n-p} R_{a(s, t)} & \text { if } 0 \leq p \leq m-n, \\
B_{1}^{p+1} \cap B_{3}^{n-q}=\bigoplus_{\substack{t \geq n-p \\
s \leq n-q}} R_{a(s, t)}, & \text { if } m-n+1 \leq p \leq n-1, \\
B_{3}^{n-q}=\bigoplus_{s \geq n-q} R_{a(s, t)}, & \text { if } n \leq p \leq m .\end{cases} \\
= & \begin{cases}M(0, n-p), & \text { if } 0 \leq p \leq m-n, \\
M(n-q, n-p), & \text { if } m-n+1 \leq p \leq n-1, \\
M(n-q, 0), & \text { if } n \leq p \leq m .\end{cases}
\end{aligned}
$$

The proof for the case $m>2 n$ is similar.

Next we prove (ii). Assume that $m$ is even and $n+1 \leq m \leq 2 n$. We recall that by Theorem 9.18 of [L], an irreducible constituent $R_{a(s, t)}$ of $S^{-\frac{m}{2},-\frac{m}{2}}\left(X^{o o}\right)$ 


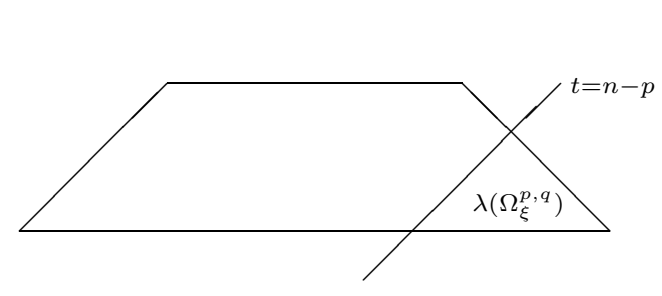

$0 \leq p \leq m-n$

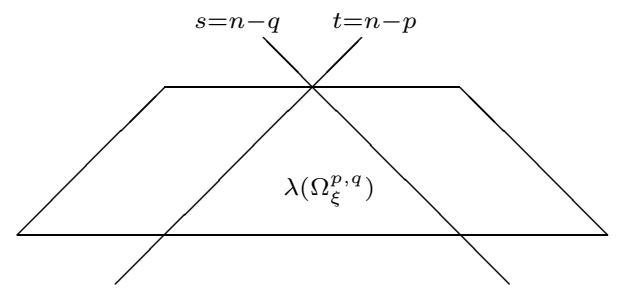

$m-n+1 \leq p \leq n-1$

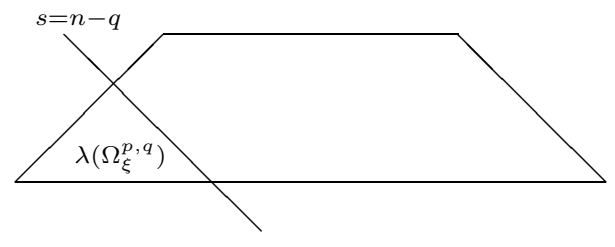

$n \leq p \leq m$

Figure 4

is unitary if and only if it is on the "highest level" or the "lowest level" of the module diagram; i.e., when $s+t=2 n-m$ or $s+t=n$. If $p q=0$, then $Q^{p q}$ corresponds to either $R_{a(n, 0)}$ or $R_{a(0, n)}$, hence is unitary. If $m-n \leq p \leq n$, then $Q_{\xi}^{p, q} \cong R_{a(n-q, n-p)}$ is unitary since $(n-q)+(n-p)=2 n-m$. It is easy to see these are all the $Q_{\xi}^{p, q}$ which are unitary.

The proof for (iii) is similar.

We shall identify the images of $\Omega_{\xi}^{p, q}$ in the module diagram of $S^{-\frac{m}{2},-\frac{m}{2}}\left(X^{o o}\right)$ or $S^{-m, 0}\left(X^{o o}\right)$. The diagrams for the cases $n+1 \leq m \leq 2 n-1$ are given in Fig. 4 . In the case $m \geq 2 n$, the module diagram for $S^{-\frac{m}{2},-\frac{m}{2}}\left(X^{o o}\right)$ or $S^{-m, 0}\left(X^{o o}\right)$ is a full triangle and the images of $\Omega_{\xi}^{p, q}$ are given in Fig. 5 .

We shall now summarize the module structure of $\Omega_{\xi}^{p, q}$ in the following theorem. Recall that if $M$ is a module for a group or algebra, then the socle of $M$ is the sum of all irreducible submodules of $M$, and is written $\operatorname{Soc}(M)$ (see [GW]). The socle series of $M$ is the ascending chain

$$
\operatorname{Soc}^{0}(M) \subseteq \operatorname{Soc}^{1}(M) \subseteq \operatorname{Soc}^{2}(M) \subseteq \cdots
$$

of submodules of $M$ defined inductively by setting $\operatorname{Soc}^{0}(M)=0$ and

$$
\operatorname{Soc}^{r+1}(M) / \operatorname{Soc}^{r}(M)=\operatorname{Soc}\left(M / \operatorname{Soc}^{r}(M)\right)
$$

for any nonnegative integer $r$.

Theorem 5.5. Let $m$ be a positive integer and $p+q=m$.

(a) If $1 \leq m \leq n$, then $\Omega_{\xi}^{p, q}$ is irreducible and unitary. In this case we have $\Omega_{\xi}^{p, q}=Q_{\xi}^{p, q}$ so that $Q_{\xi}^{p, q}$ is also unitary.

(b) If $m \geq n+1$, then $\Omega_{\xi}^{p, q}$ and $Q_{\xi}^{p, q}$ has the following structure.

(i) If we denote the preimage of a constituent $R_{a(s, t)}$ in $\lambda\left(\Omega_{\xi}^{p, q}\right)$ also by $R_{a(s, t)}$, then

$$
\Omega_{\xi}^{p, q}=\sum\left\{R_{a(s, t)}: s+t \leq n, s \geq d_{1}, t \geq d_{2}\right\}
$$

where $d_{1}=\max \{0, n-q\}$ and $d_{2}=\max \{0, n-p\}$. 

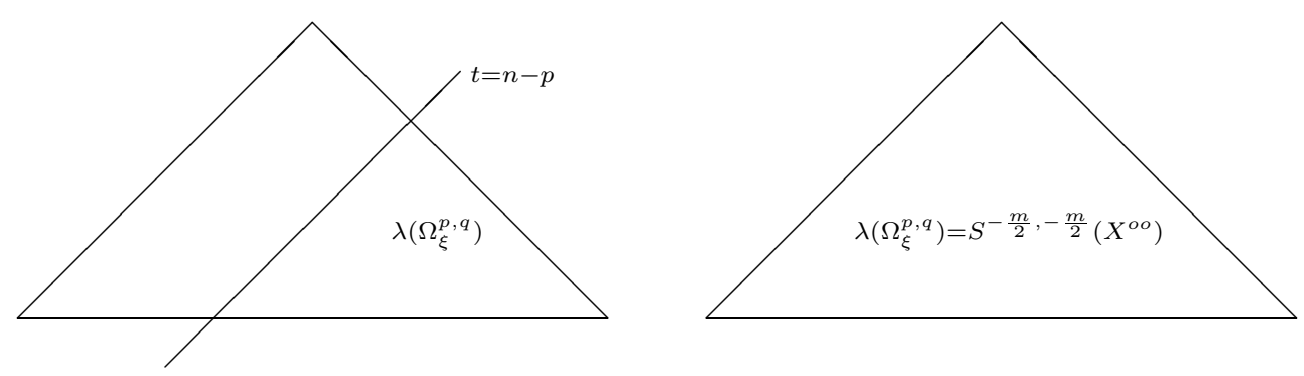

$0 \leq p \leq m-n$

$m-n+1 \leq p \leq n-1$

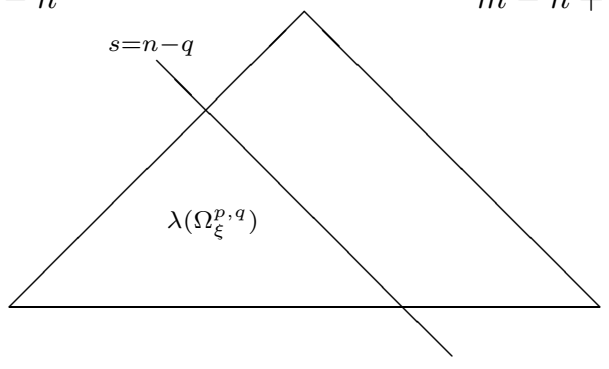

$n \leq p \leq m$

FiguRE 5

(ii) The module diagram of $\Omega_{\xi}^{p, q}$ is a (upright) triangle with $\left(n-d_{1}-d_{2}+1\right)$ rows. Consequently the socle series of $\Omega_{\xi}^{p, q}$ is given by

$\operatorname{Soc}^{j}\left(\Omega_{\xi}^{p, q}\right)=\sum\left\{R_{a(s, t)}: n-j+1 \leq s+t \leq n, s \geq d_{1}, t \geq d_{2}\right\}$

for $1 \leq j \leq n-d_{1}-d_{2}$ and $\operatorname{Soc}^{j}\left(\Omega_{\xi}^{p, q}\right)=\Omega_{\xi}^{p, q}$ for $j \geq n-d_{1}-d_{2}+1$.

Hence $\Omega_{\xi}^{p, q}$ has a socle length $n-d_{1}-d_{2}+1$.

(iii) For $0 \leq i \leq n-d_{1}-d_{2}$ and $0 \leq j \leq n-d_{1}-d_{2}-i$, set

$$
M_{i, j}=\operatorname{Soc}^{i}\left(\Omega_{\xi}^{p, q}\right) \oplus \sum_{k=0}^{j} R_{a\left(n-d_{2}-i-k, d_{2}+k\right)} .
$$

Then

$$
\begin{aligned}
0 \subseteq & M_{0,0} \subseteq M_{0,1} \subseteq \cdots \subseteq M_{0, n-d_{1}-d_{2}} \\
\subseteq & M_{1,0} \subseteq M_{1,1} \subseteq \cdots \subseteq M_{1, n-d_{1}-d_{2}-1} \\
\subseteq & \cdots \\
& \cdots \\
& \cdots \\
\subseteq & M_{n-d_{1}-d_{2}, 0}=\Omega_{\xi}^{p, q}
\end{aligned}
$$

is a composition series for $\Omega_{\xi}^{p, q}$.

(iv) $Q_{\xi}^{p, q}$ is isomorphic to $R_{a\left(d_{1}, d_{2}\right)}$. It is unitary if and only if either $n+1 \leq$ $m \leq 2 n$ and $p, q \leq n$, or $p q=0$. It is finite dimensional if and only if $p \geq n$ and $q \geq n$, and in this case, $Q_{\xi}^{p, q} \cong R_{a(0,0)}$. 


$$
R_{a\left(d_{1}, d_{2}\right)}
$$

$$
R_{a\left(d_{1}+1, d_{2}\right)} \quad R_{a\left(d_{1}, d_{2}+1\right)}
$$

$$
R_{a\left(d_{1}+2, d_{2}\right)}
$$

$$
R_{a\left(d_{1}+1, d_{2}+1\right)}
$$

$$
R_{a\left(d_{1}, d_{2}+2\right)}
$$

$R_{a\left(n-d_{2}, d_{2}\right)}$

$R_{a\left(n-d_{2}-1, d_{2}+1\right)}$

$$
R_{a\left(d_{1}+1, n-d_{1}-1\right)}
$$

$R_{a\left(d_{1}, n-d_{1}\right)}$

FiguRE 6

The positions of the constituents in the module diagram for $\Omega_{\xi}^{p, q}$ in the case $m \geq n+1$ is given in Fig. 6 .

We now consider the modules $\Omega_{\xi}^{p, q}(l)$ for $l \neq 0$ and $p+q=n$.

Proposition 5.6. Let $l$ be a nonzero integer and let $m=n$. Then

$$
\lambda\left(\Omega_{\xi}^{p, q}(l)\right)=R_{a(p, q)} \subseteq \begin{cases}I(|l|,-l) & \text { if } n \text { is even, } \\ I(|l|,-n-l) & \text { if } n \text { is odd. }\end{cases}
$$

Hence as a $U(n, n)$ module, $\Omega_{\xi}^{p, q}(l)$ is irreducible and unitary.

Proof. We shall only prove the case when $n$ is even and $l>0$ as the proofs for the other cases are similar. By Theorem 4.1, there is an embedding $\lambda: \Omega_{\xi}^{p, q}(l) \longrightarrow$ $I(l ;-l) \cong S^{\alpha, \beta}\left(X^{o o}\right)$ where $\alpha=-\left(\frac{n}{2}+l\right)$ and $\beta=-\frac{n}{2}$. Now by Proposition 2.2 and Corollary 3.6, the $K$-types in $\Omega_{\xi}^{p, q}(l)$ are of the form $\rho^{\lambda} \otimes \rho^{\lambda^{*}-l \mathbf{1}_{n}}$ where

$$
\lambda=\frac{p-q}{2} \mathbf{1}_{n}+\left(\alpha_{1}, \ldots, \alpha_{p},-\gamma_{q}, \ldots,-\gamma_{1}\right),
$$

and $\alpha_{1} \geq \cdots \geq \alpha_{p} \geq 0$ and $\gamma_{1} \geq \cdots \geq \gamma_{q} \geq l$. Observe that $\frac{p-q}{2}=-(\beta+n-p)$ and $\frac{p-q}{2}-l=\alpha+(p+1)-1$. Hence the condition on $\lambda$ is equivalent to the conditions

$$
\begin{aligned}
\lambda_{p} & \geq \frac{p-q}{2}=-(\beta+n-p), \\
\lambda_{p+1} & \leq \frac{p-q}{2}-l=\alpha+(p+1)-1 .
\end{aligned}
$$

Note that these are the conditions which define the constituent $R_{a(p, q)}$ in the space $S^{-\frac{n}{2}-l,-\frac{n}{2}}\left(X^{o o}\right)$. This shows that $\lambda\left(\Omega_{\xi}^{p, q}(l)\right)$ and $R_{a(p, q)}$ have the same collection of $K$-types. Consequently $\lambda\left(\Omega_{\xi}^{p, q}(l)\right)=R_{a(p, q)}$.

Remark 5.7. By using results from [Li], one can show in general that $\Omega_{\xi}^{p, q}(l)$ is an irreducible and unitary module of $U(n, n)$ if $p+q \leq n$ (see also Proposition 3.1 of $[\mathrm{KR} 2])$. 
We have seen in Theorem 5.5 that the structure of $\Omega_{\xi}^{p, q}$ is determined by the representation $I(m-n ; 0)$ or $I(m-n ;-m)$. Howe and Kudla have raised the following question: To what extent is the structure of $I(m-n ; 0)$ and $I(m-n ;-m)$ determined by the images of the various $\Omega_{\xi}^{p, q}$ for $p+q=m$ ? This question now has a neat answer.

Proposition 5.8. Let $m$ be an integer such that $m \geq n+1$. For $s, t \geq 0$ with $s+t \leq n$, we consider the constituent $R_{a(s, t)}$ in $I(m-n ; 0) \cong S^{-\frac{m}{2},-\frac{m}{2}}\left(X^{o o}\right)$ if $m$ is even and in $I(m-n ;-m) \cong S^{-m, 0}\left(X^{o o}\right)$ if $m$ is odd.

(i) For $s+t=n$, we have

$$
R_{a(s, t)}=\lambda\left(\Omega^{m-n+s, n-s}\right) \cap \lambda\left(\Omega^{n-t, m-n+t}\right) .
$$

(ii) For $s+t<n, R_{a(s, t)}$ is isomorphic to the quotient of

$$
\lambda\left(\Omega^{m-n+s, n-s}\right) \cap \lambda\left(\Omega^{n-t, m-n+t}\right)
$$

by

$$
\left[\lambda\left(\Omega^{m-n+s+1, n-s-1}\right) \cap \lambda\left(\Omega^{n-t, m-n+t}\right)\right]+\left[\lambda\left(\Omega^{m-n+s, n-s}\right) \cap \lambda\left(\Omega^{n-t-1, m-n+t+1}\right)\right] .
$$

Proof. We shall only prove part (ii) in the case when $m$ is even. Recall for $0 \leq$ $s, t \leq n$ and $s+t \geq 2 n-m, M(s, t)$ is a submodule of $S^{-\frac{m}{2},-\frac{m}{2}}\left(X^{o o}\right)$ and $R_{a(s, t)}$ is the constituent in $S^{-\frac{m}{2},-\frac{m}{2}}\left(X^{o o}\right)$ which occupies the tip of its module diagram. In fact,

$$
R_{a(s, t)} \cong M(s, t) /[M(s+1, t)+M(s, t+1)] .
$$

On the other hand, one observes that

$$
M(s, t)=M(s, 0) \cap M(0, t) .
$$

Hence by Proposition 5.4,

$$
M(s, t)=\lambda\left(\Omega^{m-n+s, n-s}\right) \cap \lambda\left(\Omega^{n-t, m-n+t}\right) .
$$

Consequently, $R_{a(s, t)}$ is isomorphic to the quotient of

$$
\lambda\left(\Omega^{m-n+s, n-s}\right) \cap \lambda\left(\Omega^{n-t, m-n+t}\right)
$$

by

$$
\left[\lambda\left(\Omega^{m-n+s+1, n-s-1}\right) \cap \lambda\left(\Omega^{n-t, m-n+t}\right)\right]+\left[\lambda\left(\Omega^{m-n+s, n-s}\right) \cap \lambda\left(\Omega^{n-t-1, m-n+t+1}\right)\right] .
$$

\section{Gelfand-Kirillov Dimensions of irReducible Constituents}

Recall that each irreducible constituent $R_{a(s, t)}$ of $S^{\alpha, \beta}\left(X^{o o}\right)$ corresponds to a subquotient of $S^{\alpha, \beta}\left(X^{o o}\right) \cong I(s ; \nu)$. We shall abuse notation and denote this subquotient also by $R_{a(s, t)}$. The purpose of this section is to determine the GelfandKirillov dimension of the subquotient $R_{a(s, t)}$.

We first recall the definition of Gelfand-Kirillov dimension for a finitely generated $\mathfrak{U}(\mathfrak{g})$ module $W$, where $\mathfrak{g}$ is a Lie algebra over $\mathbb{C}$ and $\mathfrak{U}(\mathfrak{g})$ is the enveloping algebra of $\mathfrak{g}$ (see $[\mathrm{V}])$. Choose a finite dimensional subspace $W_{0}$ so that $W=\mathfrak{U}(\mathfrak{g}) W_{0}$. For each positive integer $k$, let $\mathfrak{U}(\mathfrak{g})_{k}$ be the subspace of $\mathfrak{U}(\mathfrak{g})$ spanned by products of at most $k$ elements in $\mathfrak{g}$. We set $d_{W, W_{0}}(k)=\operatorname{dim} \mathfrak{U}_{k}(\mathfrak{g}) W_{0}$. Then there is a polynomial $\phi$ of degree at most dim $\mathfrak{g}$ such that $d_{W, W_{0}}(k)=\phi(k)$ for large $k$. Moreover the 
leading term which we write as $\frac{c(W)}{(\operatorname{Dim} W) !} k^{\operatorname{Dim} W}$ is independent of the choice of $W_{0}$. $\operatorname{Dim} W$ is called the Gelfand-Kirillov dimension of $W$.

Recall that $G=U(n, n)$ and $K=U(n) \times U(n)$, and let $\mathfrak{g}=\mathfrak{u}(n, n)_{\mathbf{C}}$. Let $V$ be one of the subquotient $R_{a(s, t)}$ of $S^{\alpha, \beta}\left(X^{o o}\right)$. Then $V$ admits the $K$-isotypic decomposition

$$
\left.V\right|_{K} \simeq \sum_{\lambda \in R} V_{\lambda}
$$

where $R$ is the subset of $\Lambda_{n}^{+}$given in (5.1) and (5.3). Now for each $\lambda=\left(\lambda_{1}, \ldots, \lambda_{n}\right) \in$ $\Lambda_{n}^{+}$, we let $|\lambda|=\left|\lambda_{1}\right|+\cdots+\left|\lambda_{n}\right|$. Let $\lambda_{0}$ be such that $\left|\lambda_{0}\right|$ is minimum among all $\lambda \in R$. Let

$$
V_{0}=\sum_{\lambda \in R,|\lambda|=\left|\lambda_{0}\right|} V_{\lambda}
$$

and

$$
V_{k}=\sum_{\lambda \in R,|\lambda|=\left|\lambda_{0}\right|+k} V_{\lambda}
$$

for a positive integer $k$. Now $\mathfrak{u}(n, n)$ admits a Cartan decomposition $\mathfrak{u}(n, n)=$ $\mathfrak{k} \oplus \mathfrak{p}$, where $\mathfrak{k}$ is the Lie algebra of $K$, and $\mathfrak{p}=\left\{\left(\begin{array}{cc}0 & a \\ (\bar{a})^{t} & 0\end{array}\right): a \in \mathfrak{g l}_{n}(\mathbb{C})\right\}$. From the transition formulas (Propositions 5.7 and 5.15 of [L]), we see that

$$
\mathfrak{p}\left(V_{0}\right)=V_{1}, \quad \text { and } \quad \mathfrak{p}\left(V_{j}\right)=V_{j-1} \oplus V_{j+1}, \quad(j>1) .
$$

It follows from this that $\mathfrak{U}_{k}(\mathfrak{g}) V_{0}=\sum_{j \leq k} V_{j}$. Therefore if $\operatorname{dim} V_{k}$ is a polynomial in $k$ of degree $d-1$, then the Gelfand-Kirillov dimension of $V$ is equal to $d$.

We now discuss some general properties of generating functions.

Lemma 6.1. Let $\phi$ be a polynomial of degree $d$, then

$$
\sum_{k \geq 0} \phi(k) t^{k}=\frac{F(t)}{(1-t)^{d+1}}
$$

where $F$ is a polynomial with $F(1) \neq 0$.

Proof. If we write $\phi(x)=a_{0}+a_{1}(x+1)+\ldots+a_{d}(x+1) \cdots(x+d)$, where $a_{d} \neq 0$, then the lemma follows from the identity

$$
\sum_{k \geq 0}(k+1) \cdots(k+d) t^{k}=\frac{d !}{(1-t)^{d+1}} .
$$

Note that in fact, $F(1)=a_{d} \cdot d$ !. In particular, $F(1)$ is positive for $\phi(k)=k^{d}$.

Lemma 6.2. Let $\phi\left(\alpha_{1}, \ldots, \alpha_{p}\right)$ be a polynomial of total degree $d$. Write $\phi=\phi_{h}+\phi^{\prime}$, where $\phi_{h}$ is homogeneous of total degree $d$ and $\phi^{\prime}$ is of total degree less than or equal to $d-1$. Suppose that the coefficients of $\phi_{h}$ are non-negative. Then for any given positive integers $c_{1}, \ldots, c_{p}$, we have

$$
\sum_{\alpha_{1}, \ldots, \alpha_{p} \geq 0} \phi\left(\alpha_{1}, \ldots, \alpha_{p}\right) t^{c_{1} \alpha_{1}+\ldots+c_{p} \alpha_{p}}=\frac{P(t)}{(1-t)^{d+p} Q(t)},
$$

where $P$ and $Q$ are polynomials with $P(1)>0, Q(1)>0$. 
Proof. Note that

$$
\sum_{\alpha_{1}, \ldots, \alpha_{p} \geq 0} \alpha_{1}^{k_{1}} \cdots \alpha_{p}^{k_{p}} t_{1}^{c_{1} \alpha_{1}} \cdots t_{p}^{c_{p} \alpha_{p}}=\prod_{1 \leq l \leq p}\left(\sum_{\alpha_{l} \geq 0} \alpha_{l}^{k_{l}} t_{l}^{c_{l} \alpha_{l}}\right)
$$

By Lemma 6.1 , for each $1 \leq l \leq p$, we have

$$
\sum_{\alpha_{l} \geq 0} \alpha^{k_{l}} t_{l}^{c_{l} \alpha_{l}}=\frac{F_{l}\left(t_{l}^{c_{l}}\right)}{\left(1-t_{l}^{c_{l}}\right)^{k_{l}+1}}
$$

where $F_{l}$ is a polynomial with $F_{l}(1)>0$. Hence if we put $t_{1}=\ldots=t_{p}=t$, then the expression in (6.1) is equal to

$$
\sum_{\alpha_{1}, \ldots, \alpha_{p} \geq 0} \alpha_{1}^{k_{1}} \cdots \alpha_{p}^{k_{p}} t^{c_{1} \alpha_{1}+\cdots c_{p} \alpha_{p}}=\frac{F_{1}\left(t^{c_{1}}\right) \cdots F_{p}\left(t^{c_{p}}\right)}{\left(1-t^{c_{1}}\right)^{\left(k_{1}+1\right)} \cdots\left(1-t^{c_{p}}\right)^{\left(k_{p}+1\right)}},
$$

where $F_{l}(1)$ is positive, for $1 \leq l \leq p$. The lemma clearly follows from this.

Lemma 6.3. Under the hypothesis of Lemma 6.2,

$$
\psi(k)=\sum_{\substack{\alpha_{1}, \ldots, \alpha_{p} \geq 0 \\ c_{1} \alpha_{1}+\ldots+c_{p} \alpha_{p}=k}} \phi\left(\alpha_{1}, \ldots, \alpha_{p}\right)
$$

is a polynomial in $k$ of degree $d+p-1$.

Proof. First note that the sum defining $\psi(k)$ is a finite sum, so $\psi(k)$ is a polynomial in $k$. Let $e$ be its degree. We know by Lemma 6.1 that

$$
\sum_{k \geq 0} \psi(k) t^{k}=\frac{F(t)}{(1-t)^{e+1}}
$$

where $F$ is a polynomial with $F(1) \neq 0$. On the other hand, by Lemma 6.2 , we have

$$
\sum_{k \geq 0} \psi(k) t^{k}=\sum_{\alpha_{1}, \ldots, \alpha_{p} \geq 0} \phi\left(\alpha_{1}, \ldots, \alpha_{p}\right) t^{c_{1} \alpha_{1}+\cdots c_{p} \alpha_{p}}=\frac{P(t)}{(1-t)^{d+p} Q(t)}
$$

where $P$ and $Q$ are polynomials with $P(1)>0, Q(1)>0$. By comparing the order of the pole at $t=1$, we obtain $e+1=d+p$.

We are now ready to compute the Gelfand-Kirillov dimensions of $R_{a(s, t)}$. 
Theorem 6.4. The Gelfand-Kirillov dimension of $R_{a(s, t)}$ is equal to $(s+t)(2 n-$ $s-t)$.

Proof. We shall only examine the case when $\alpha+\beta+n-1 \leq 0$. The case when $\alpha+\beta+n-1>0$ is similar. We know that $V_{\lambda} \subseteq R_{a(s, t)}$ if and only if

$$
\begin{gathered}
\lambda_{1} \geq \ldots \geq \lambda_{s} \geq \alpha+s, \\
\alpha+s \geq \lambda_{s+1} \geq \ldots \geq \lambda_{n-t} \geq-(\beta+t), \\
-(\beta+t) \geq \lambda_{n-t} \geq \ldots \geq \lambda_{n} .
\end{gathered}
$$

We make the following change of variables:

$$
\begin{aligned}
& \mu_{j}=\lambda_{j}-\lambda_{j+1}, \quad 1 \leq j \leq s-1, \\
& \mu_{s}=\lambda_{s}-(\alpha+s), \\
& \mu_{j}=\lambda_{j}, \quad s+1 \leq j \leq n-t, \\
& \mu_{j}=\lambda_{2 n-j-t}-\lambda_{2 n-j-t+1}, \quad n-t+1 \leq j \leq n-1, \\
& \mu_{n}=-(\beta+t)-\lambda_{n-t+1} .
\end{aligned}
$$

We shall now write $V(\mu)$ for $V_{\lambda}$. Observe that $\lambda \in R_{a(s, t)}$ and $|\lambda|=k$ imply that

$$
\begin{aligned}
& \mu_{1}, \ldots, \mu_{s}, \mu_{1}, \ldots, \mu_{t} \geq 0, \\
& \alpha+s \geq \mu_{s+1} \geq \ldots \geq \mu_{n-t} \geq-(\beta+t), \\
& k-k_{0} \leq \mu_{1}+2 \mu_{2}+\ldots+s \mu_{s}+\mu_{n-t+1}+2 \mu_{n-t+2}+\ldots+t \mu_{n} \leq k+k_{0},
\end{aligned}
$$

for some fixed integer $k_{0}$. Conversely,

$$
\begin{aligned}
& \mu_{1}, \ldots, \mu_{s}, \mu_{1}, \ldots, \mu_{t} \geq 0, \\
& \alpha+s \geq \mu_{s+1} \geq \ldots \geq \mu_{n-t} \geq-(\beta+t), \\
& \mu_{1}+2 \mu_{2}+\ldots+s \mu_{s}+\mu_{n-t+1}+2 \mu_{n-t+2}+\ldots+t \mu_{n}=k
\end{aligned}
$$

will imply that $\lambda \in R_{a(s, t)}$ and $k-k_{1} \leq|\lambda| \leq k+k_{1}$ for some fixed integer $k_{1}$. Therefore when $k$ is sufficiently large,

$$
\sum_{\substack{\lambda \in R_{a(s, t)} \\|\lambda|=k}} \operatorname{dim} V_{\lambda}
$$

will have the same degree as

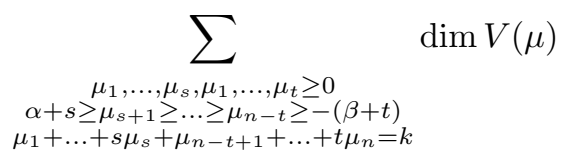

as a polynomial in $k$.

By the Weyl dimension formula, we have

$$
\operatorname{dim} V_{\lambda}=\left(\operatorname{dim} \rho^{\lambda}\right)^{2}=\prod_{1 \leq i<j \leq n} \frac{\left(\lambda_{i}-\lambda_{j}+j-i\right)^{2}}{(j-i)^{2}} .
$$

In the new variables, we see that $\operatorname{dim} V(\mu)$ is a polynomial in $\mu_{1}, \ldots, \mu_{s}, \mu_{n-t+1}, \ldots$, $\mu_{n}$ of total degree

$$
\begin{aligned}
& 2[(n-1)+\ldots+(n-s)+\underbrace{t+\ldots+t}_{n-s-t \text { times }}+(t+1)+\ldots+1] \\
& =(s+t)[2 n-(s+t)-1] .
\end{aligned}
$$


Moreover the relationship between $\lambda$ and $\mu\left(\lambda_{1}-\lambda_{3}=\mu_{1}+\mu_{2}\right.$, etc. $)$ implies that the condition on the coefficients of the homogeneous part of $\operatorname{dim} V(\mu)$ specified in Lemma 6.2 is satisfied. Therefore by Lemma 6.3, we see that

$$
\sum_{\substack{\mu_{1}, \ldots, \mu_{s}, \mu_{1}, \ldots, \mu_{t} \geq 0 \\ \alpha+s \geq \mu_{s+1} \geq \ldots \geq \mu_{n-t} \geq-(\beta+t) \\ \mu_{1}+\ldots+s \mu_{s}+\mu_{n-t+1}+\ldots+t \mu_{n}=k}} \operatorname{dim} V(\mu)
$$

is a polynomial in $k$ of degree

$$
(s+t)[2 n-(s+t)-1]+(s+t)-1=(s+t)[2 n-(s+t)]-1 .
$$

Thus

$$
\sum_{\substack{\lambda \in R_{a(s, t)} \\|\lambda|=k}} \operatorname{dim} V_{\lambda}
$$

is a polynomial in $k$ of the same degree. It follows from this and the discussion before Lemma 6.1 that the Gelfand-Kirillov dimension of $R_{a(s, t)}$ is equal to $(s+t)[2 n-(s+t)]$.

We recall that all the constituents $R_{a(s, t)}$ in $S^{\alpha, \beta}\left(X^{o o}\right)$ with $s+t$ fixed are on the same "level" of the module diagram of $S^{\alpha, \beta}\left(X^{o o}\right)$. Now by Theorem 6.4 , we see that the Gelfand-Kirillov dimension of $R_{a(s, t)}$ is a function of $s+t$. Hence all the constituents occupying the same level in the module diagram have the same Gelfand-Kirillov dimension. Here we consider an example. Assume that $\alpha+\beta+$ $n-1 \leq-(n+1)$. Then the module diagram of $S^{\alpha, \beta}\left(X^{o o}\right)$ is a (complete) upright triangle with $n+1$ rows (see Fig. 5 or Fig. 11 of $[\mathrm{L}]$ ). The top row consists of only the constituent $R_{a(0,0)}$ which has Gelfand-Kirillov dimension 0; that is, $R_{a(0,0)}$ is finite dimensional. The next row consists of the constituents $R_{a(1,0)}$ and $R_{a(0,1)}$. Each of them has Gelfand-Kirillov dimension $2 n-1$. The Gelfand-Kirillov dimensions of the constituents increase when we move from one level of the module diagram to the next lower level. The lowest level consists of the constituents $R_{a(n, 0)}$, $R_{a(n-1,1)}, \ldots, R_{a(0, n)}$. They have the largest possible Gelfand-Kirillov dimension among the $R_{a(s, t)}$ 's, which is $n^{2}$.

\section{REFERENCES}

[Ad] J. Adams, The theta correspondence over $\mathbb{R}$, preprint.

[Al] J. Alperin, Diagrams for modules, J. Pure Appl. Algebra 16 (1980), 111-119. MR 81h: 16047

[Ba] V. Bargmann, On a Hilbert space of analytic functions and an associated integral transform, I, Comm. Pure Appl. Math. 14 (1961), 187-214. MR 28:486

[BGG] I. N. Bernstein, I. M. Gelfand and S. I. Gelfand, Model of representations of Lie groups, Sel. Math. Sov. 1 (1981), 121-142. MR 54:2884

[Fo] G. Folland, "Harmonic analysis in phase space," Ann. of Math. Stud. 122, Princeton Univ. Press, Princeton, 1989. MR 92k:22017

[Ge] S. Gelbart, Examples of dual pairs, in "Automorphic forms, representations and $L$ functions," Proc. Sympos. Pure Math., Vol. 33, Part 1, pp. 287-296, Amer. Math. Soc., Providence, RI, 1979. MR 81f:22035

[GW] K. Goodearl and R. Warfield, "An introduction to noncommutative rings," London Mathematical Society Student Texts, Vol. 16, Cambridge Univ. Press, Cambridge, 1989. MR 91c:16001

[H1] R. Howe, Remarks on classical invariant theory, Trans. Amer. Math. Soc. 313(1989), 539570. MR 90h:22015a 
[H2] R. Howe, Transcending classical invariant theory, J. Amer. Math. Soc. 2(1989), 535-552. MR 90k:22016

[H3] R. Howe, Dual pairs in physics: "Harmonic oscillators, photons, electrons, and singletons," Lectures in Appl. Math., Vol 21, pp. 179-206, Amer. Math. Soc., Providence, RI, 1985. MR 86i: 22036

[H4] R. Howe, $\theta$-series and invariant theory, in "Automorphic forms, representations and $L$ functions," Proc. Sympos. Pure Math., Vol 33, Part 1, pp.275-286, Amer. Math. Soc., Providence, RI, 1979. MR 81f:22034

[H5] R. Howe, Oscillator representation: algebraic and analytic preliminaries, preprint.

[HT] R. Howe and E.C. Tan, "Non-Abelian Harmonic Analysis: Applications of $S L(2, \mathbb{R})$," Universitext, Springer-Verlag, New York, 1992. MR 93f:22009

[J1] K. Johnson, Degenerate principal series and compact groups, Math. Ann. 287(1990), 703718. MR 91h:22028

[J2] K. Johnson, Degenerate principal series on tube type domains, Contemp. Math. 138(1992), 175-187. MR 94b:22010

[KR1] S. Kudla and S. Rallis, Degenerate principal series and invariant distributions, Israel J. Math. 69(1990), 25-45. MR 91e:22016

[KR2] S. Kudla and S. Rallis, Ramified degenerate principal series representations for $S p(n)$, Israel J. Math. 78(1992), 209-256. MR 94a:22035

[KRS] S. Kudla, S. Rallis and D. Soudry, On the degree $5 \mathrm{~L}$-function for Sp(2), Invent. Math. 107(1992), 483-541. MR 93b:11061

[Ku1] S. Kudla, Seesaw dual reductive pairs, Progr. Math. 46(1983), 244-268. MR 86b:22032

[Ku2] S. Kudla, Splitting metaplectic covers of dual reductive pairs, Israel J. Math. 87(1994), 361-401. MR 95h:22019

[KV] M. Kashiwara and M. Vergne, On the Segal-Shale-Weil representations and harmonic polynomials, Invent. Math. 44 (1978),1-47. MR 57:3311

[L] S. Lee, On some degenerate principal series representations of $U(n, n)$, J. of Funct. Anal. 126(1994), 305-366. MR 95j:22023

[Li] J. Li, Singular unitary representations of classical groups, Invent. Math. 97 (1989), 237255. MR 90h:22021

[S1] S. Sahi, Unitary representations on the Shilov boundary of a symmetric tube domain, in "Representations of Groups and Algebras," Contemp. Math. 145(1993), 275-286, Amer. Math. Soc., Providence. MR 94e:22029

[S2] S. Sahi, Jordan algebras and degenerate principal series, J. reine angew Math. 462 (1995), 1-18. MR 96d:22022

[V] D. Vogan, Gelfand-Kirillov Dimension for Harish-Chandra Modules, Invent. Math. 48(1978), 75-98. MR 58:22205

[Wey] H. Weyl, "The classical groups," Princeton University Press, Princeton, N.J., 1946.

[Wei] A. Weil, Sur certains groupes d'operateurs unitaires, Acta Math. 111(1964). 143-211. MR 29:2324

[Zha] G. Zhang, Jordan algebras and generalized principal series representation, Math. Ann. 302(1995), 773-786. MR 97h:22012

[Zhe] D. P. Zhelobenko, "Compact Lie Groups and their Representations," Translations of Mathematical Monographs, vol.40, Amer. Math. Soc., 1973. MR 57:12776a

[Zhu] C. Zhu, Invariant distributions of classical groups, Duke Math. J. 65(1)(1992), 85-119. MR 92k:22022

Department of Mathematics, National University of Singapore, 10 Kent Ridge CresCent, Singapore 119260, Republic of Singapore

E-mail address: matleest@leonis.nus.edu.sg

Department of Mathematics, National University of Singapore, 10 Kent Ridge CresCent, Singapore 119260, Republic of Singapore

E-mail address: matzhucb@leonis.nus.edu.sg 